\title{
A computational approach to flame hole dynamics using an embedded manifold approach
}

\author{
R. Knaus*, C. Pantano \\ Department of Mechanical Science and Engineering, University of Illinois at Urbana-Champaign, Urbana, IL \\ 61801, USA
}

\begin{abstract}
We present a new numerical algorithm for tracking the evolution of flame holes in diffusion flames. The key element is the solution of an evolution equation for a flame state field defined on a complex moving surface. The surface itself can evolve in time and is defined implicitly as a level set of an associated Cartesian scalar field. The surface coordinates, or parameterization, do not need to be determined explicitly. Instead, the numerical method employs an embedding technique where the evolution equation is extended to the Cartesian space. In our application, the flame state field represents the chemical activity state of a diffusion flame; i.e. quenched and burning regions of the flame surface. We present a formulation that describes the formation, propagation, and growth of flames holes using edge-flame modeling in laminar and turbulent diffusion flames. The evolution equation is solved using a high-order finite-volume WENO method and a new extension algorithm defined in terms of propagation PDEs. The complete algorithm is demonstrated by tracking the dynamics of flame holes in a turbulent reacting shear layer and its applicability is also demonstrated in a turbulent reacting lifted jet simulation.
\end{abstract}

Keywords: Flame hole dynamics, Embedded manifold, Extinction/reignition, Turbulent diffusion flames, High-order WENO, Non-conservative systems

\section{Introduction}

The numerical solution of partial differential equations on surfaces is an area of research with a variety of applications such as image processing, geometry, physiology, solidification, gravitation, and fluid mechanics (see Ratz and Voigt [1]). The problem of evolution of a field constrained to a surface can be described in terms of surface differential operators (gradient, divergence, etc.) if a parametric description of the surface (e.g., $\mathbf{z}=\left(z_{1}, z_{2}\right)$ ) is available. Unfortunately, it is difficult and computationally expensive to employ this approach for a general surface, where even creating a clean surface mesh might be difficult [1]. Additionally, frequent re-meshing may be needed if the surface evolves in time, and particularly if the surface can develop topological changes. An alternative approach to solving PDEs on surfaces using the parametric coordinates is to embed the surface in the three-dimensional Cartesian space. This requires a transformation of the surface

* Corresponding author

Email addresses: rknaus2@illinois.edu (R. Knaus), cpantano@illinois.edu (C. Pantano) 
PDE to an equivalent volumetric PDE that allows one to solve the latter using customary Cartesian operators.

The heart of an embedding method is an extension operator by which the surface field is extended smoothly throughout the Cartesian space. Surface data is propagated normal to isosurfaces of an embedding function that implicitly defines the surface. These methods can be classified into geometrical and differential equation-based methods. Introduced by Bertalmı et al. [2], differential equation-based methods construct a PDE defining the extension operation in the embedding space. The solution of this equation has the property that the extended surface data in the Cartesian domain is normal to the isosurfaces of the embedding function. Cartesian discretizations can then be applied in the embedding space to solve the surface PDE on the implicit surface. This method has been applied to a wide variety of problems $[1,2,3]$ and has been used for solving surface advection problems on evolving surfaces $[4,5]$. The Cartesian space data structures (rather than the intrinsic coordinates of the complex surface) result generally in faster execution. In addition, to minimize computational cost, the embedding PDE is typically only solved in a narrow band around the surface. However, solving the embedding PDE requires the imposition of artificial boundary conditions at the boundary of the band in which the embedding PDE is solved [3]. If instead we seek to solve the embedding PDE in a much larger band around the surface, the artificial imposition of boundary conditions is not an issue and this type of method does not have difficulty with extending surface data far away from the surface. In the geometrical "closest point method" $[6,7,8]$, surface data is propagated along the local normal direction by use of a closest point extension. Values of the extended surface field at grid points in the embedding Cartesian space are set to be equal to the value of the surface field evaluated at the point on the surface that is closest to the Cartesian grid point. This is implemented by determining the closest point on the surface relative to a grid point through a closest point transform and then interpolating the value of the variable at that point from the data in the Cartesian grid surrounding it. The closest point extension has the desired property that the intrinsic surface gradient operator (and divergence operator) is the same as the Cartesian gradient of the extended field when evaluated at the surface and does not require the imposition of artificial boundary conditions. The method can also be constructed to be high-order accurate. For instance, the orthogonal gradient radial basis function method of Piret [9] uses a closest point representation with the surface approximated using a radial basis function approach to obtain spectral accuracy. It has been successful in solving diffusion and advection equations [7] and has been modified to efficiently solve problems on a moving surface by Leung et al. [10]. The grid-based particle method by Leung et al. [10] reconstructs the interface through a local least squares approximation. This allows the computation of the closest point transform, which is used to apply the closest point extension. The method tracks the interface motion by using a Lagrangian particle tracking method, using the movement of the surface to update the closest point extension. This type of method allows for the efficient computation of the closest point method for the case of an evolving surface. Finding the closest point to a surface, however, becomes increasingly difficult as the distance from the surface increases. The closest point function can be costly to compute and multivalued. As previously stated, PDE-based methods do not have problematic behavior far from the surface.

The main contribution of the present paper is the development of a hybrid closest point and PDEbased Cartesian embedding method applied to a moving surface to model a dynamically-complex turbulent combustion problem. Turbulent diffusion flames experience velocity gradients (strain rate) that vary in space and time, increasing in magnitude with increasing Reynolds number. Since the combustion conversion rate is limited by the fixed chemical time scales of the elementary reactions 
that describe the combustion of each fuel-oxidizer mixture, eventually combustion is unable to adjust to fast flow time scales and the reaction is partially and locally quenched. The quenching or extinction process starts at those locations where the rate of heat release, which sustains the high temperature of the combustion, is unable to balance the rate at which the flow extracts heat from the reaction zone $[11,12]$. The flame ceases to exist once the temperature drops sufficiently bellow the extinction temperature of the flame (all remnant temperature and chemical products diffuse quickly away and radicals recombine into stable, practically inert, species). If the Reynolds number keeps increasing, for example by increasing the velocity in a jet flame, extinction will propagate and quench the flame globally (not just locally or transitorily). The process by which high strain rates quench the flame is called extinction while the healing of a quenched zone, by advection or heating to more favorable regions is termed reignition. These locally quenched regions of a flame are called here "flame holes"; although the extinction zones can have arbitrary shapes even resembling strips or islands $[13,14]$. After the development of the flame hole, the quenched region grows, shrinks, merges, splits, and changes shape depending on the evolution of the flame rim.

Computationally modeling all these flow/chemistry interactions at high Reynolds numbers is prohibitively expensive using first-principle methods, i.e. resolving all fields. The transport and reaction of each chemical species needs to be computed and the number of species is large, ranging from tens to hundreds of species in reduced and full chemical mechanisms, respectively. More importantly, in turbulent reacting flows of interest for flame hole dynamics, the reaction zone thickness is typically many times smaller than that required to resolve the turbulence [15]. In the absence of extinction, one well-established modeling approach is to represent the mixture as an ensemble of thin reaction zones called flamelets $[16,17,18]$. The reaction takes place near the stoichiometric surface, where fuel and oxidizer meet in stoichiometric proportions. Flamelets are based on an asymptotic analysis technique (not based on ad-hoc modeling concepts). The procedure consists in identifying the small flame thickness $\delta$ over which all reactions take place and separating the internal layer where these reactions are important, which is governed by

$$
-\frac{\partial}{\partial \eta}\left(\rho D_{i} \frac{\partial Y_{i}}{\partial \eta}\right)=\omega_{i}(\vec{Y}, T)
$$

where $Y_{i}$ denotes the species mass fractions, $T$ the temperature, $\omega_{i}$ the reaction rates of species $i$, and $\eta$ is an inner spatial coordinate in the reaction zone along the normal coordinate to the flame sheet (from the perspective of the outer non-reactive solution). The technical procedure is quite complicated and can be found in [19] with full details. A predecessor of the formal complete asymptotic theory is due to Peters [17], who identified a simplification to the governing equations of the internal layer by introducing a mixture fraction $Z$ (which is conserved) as an intermediate and allows Eq. (1) to be written as

$$
-\frac{1}{2} \rho \chi \frac{\partial^{2} Y_{i}}{\partial Z^{2}}=\omega_{i}(\vec{Y}, T)
$$

where $\chi$ denotes the mixture fraction rate of dissipation, see Eq. (5) below, and $\rho$ denotes density (here, for simplicity, we are not listing the energy equation which has to be solved simultaneously with Eq. (1) or Eq. (2)). The solutions of Eq. (2), $\left\{Y_{i}^{\mathrm{f}}, T^{\mathrm{f}}\right\}$, are parameterized as a function of $Z$ and $\chi$; the latter varies in space and time and allows the flame to respond (albeit instantaneously) to the changing conditions of the flow. The combustion literature refers to Eq. (2) as the steady flamelet equations, although this nomenclature is partially misleading because the flamelet does vary in time parametrically through $\chi(\vec{x}, t)$. But, the flamelet concept is inapplicable when the flame is quenched 
because there is no flame at those locations. Then, the solutions of Eq. $(2),\left\{Y_{i}^{\mathrm{m}}, T^{\mathrm{m}}\right\}$, are linear in $Z$ (because of the zero reaction rate) and represent a purely mixing state of the gas. Therefore, in principle, Eq. (2) represents both burning and fully quenched states. Modeling turbulent diffusion flames using the solutions of this equation would be accurate, by turning on or off the flamelet depending on $\chi$, if it was not for another experimental observation, namely, that the boundary that separates a flame from a quenched region has its own structure. It is found that the flame hole boundary propagates at its own speed and it has a rather complex multidimensional structure (at least nominally two dimensional); see experiments [20] and direct numerical simulations [21, 22] among many others. The flame boundary structure is a proper type of flame called an edge flame and it has mixed propagation properties, as those of a premixed flame (with a speed that we denote by $V_{e}$ ), and strain-rate dependent burning properties, as those of a diffusion flame [23, 24, 25].

The generalization of the flamelet modeling concept to broken flamelets is called flame hole dynamics, originally introduced by Dold et al. [26, 27] and used preliminary by [28] and [29]; see figure 1. It is a physically-based enhancement that incorporates the physics of the flame boundary using edge flame physics to model broken flamelets. This modeling approach removes the need for detailed calculation of the advection-diffusion-reaction problem that is tightly coupled in the edge flame. The only information required in this modeling approach is the edge flame velocity function, $V_{e}(\chi)$, as well as the orientation of the flame boundary curve, since the edge flame is supposed to propagate normal to the flame rim. Time-dependent effects, such as unstationary response of the edge flame to time variations of mixture composition, strain rate, etc., are not incorporated at this level of closure, but they could be included in future, extended models, if deemed necessary. In the end, one needs to solve an evolution equation for a flame state field on the moving stoichiometric surface, and here is where the Cartesian embedding methods play a crucial role.

A concluding remark concerns the applicability of flame hole dynamics to general turbulent diffusion flames, where several phenomena may be present simultaneously. As described above, this is a closure description that extends the applicability of the thin flamelet model to accommodate extinction according to flow-combustion physics principles based on edge flame dynamics. This is relevant in lifted jet diffusion flames and in the vicinity of the high-strain region of such flames and other situations where no other interfering processes take place. There are some things that flame hole dynamics cannot do, e.g.: forced ignition or autoignition, thick reaction zones (because flamelets fail there as well), reignition from side heating [30] (although it could be extended to this case). Therefore, the flame hole dynamics closure description has to be used judiciously and it is not a single modeling approach for all the physics in turbulent diffusion flames.

\section{Kinematic and dynamic aspects of flame hole modeling}

The flame hole dynamics description of extinction/reignition processes in non-premixed combustion assumes a thin reaction zone residing near the stoichiometric surface; sufficiently thin that it can be considered as a mathematical surface from the point of view of the hydrodynamics coupling. This surface is defined implicitly in terms of the mixture fraction field, $Z(\mathbf{x}, t)$, according to

$$
\Sigma_{\mathrm{st}}=\left\{\mathrm{x}: Z(\mathbf{x}, t)=Z_{\mathrm{st}}\right\}
$$

where $t$ denotes time, $Z_{\text {st }}$ is a constant that depends on the stoichiometry of the system and $Z(\mathbf{x}, t)$, which is bounded between zero and one, obeys a partial differential equation independent of the combustion chemistry details, given by

$$
\rho \frac{D Z}{D t}=\nabla \cdot\left(\rho D_{\operatorname{mix}} \nabla Z\right)
$$




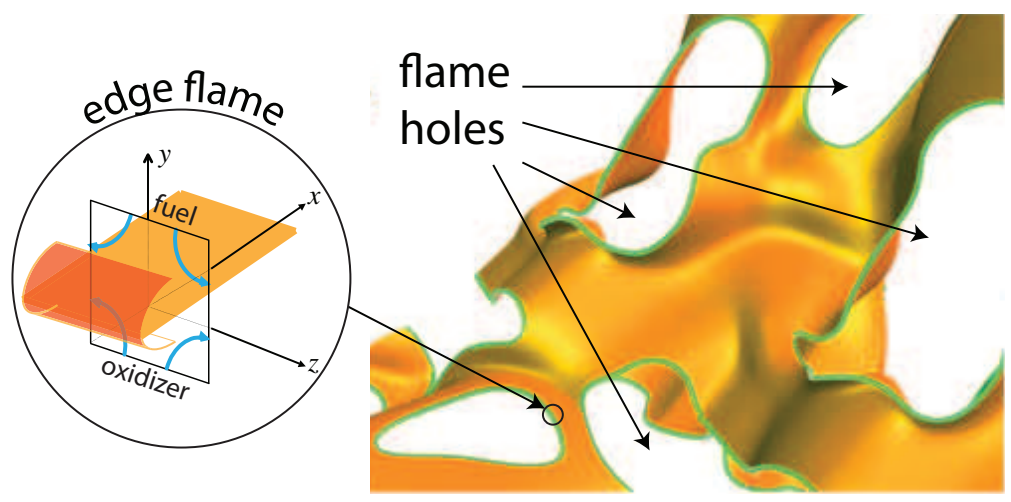

Figure 1: Flame holes in a turbulent diffusion flame [21].

where $D / D t$ denotes the material derivative, $\rho$ is the density and $D_{\text {mix }}$ denotes the average diffusivity of the mixutre, which is set equal to the thermal diffusivity $D_{\text {mix }}=\lambda / \rho c_{p}$, where $\lambda$ is the average thermal conductivity and $c_{p}$ is average specific heat at constant of the mixture; see [31]. In a leading order description, where unstationary effects are neglected, the flame strength (see [31]) responds instantaneously according to the value of the scalar dissipation, defined by

$$
\chi(\mathbf{x}, t)=2 D_{\text {mix }} \nabla Z \cdot \nabla Z .
$$

The flame will locally extinguish and form a flame hole if the scalar dissipation exceeds the quenching value, denoted by the constant $\chi_{\mathrm{q}}$ (which is well defined for each particular reacting system). The term "flame hole" here is used generally to denote local extinction regions and it is used to refer to general non-circular holes with an arbitrarily shaped boundary, $\Gamma_{i}$, where $i$ is a hole index number (assuming the holes are countable entities). The location of an isolated flame hole boundary (FHB) is denoted by its position vector $\mathbf{r}_{i}(s, t)$ with $s$ being an arc-length parameterization of the FHB. The tangent vector to the FHB is defined through $\mathbf{r}_{i}(s, t)$, according to

$$
\mathbf{t}=\frac{\partial \mathbf{r}_{i}}{\partial s}
$$

and the normal to the FHB curve is the cross product, $\mathbf{m}=\mathbf{n} \times \mathbf{t}$. By convention we chose $s$ such that $\mathbf{m}$ points in the direction of the burning region in the corresponding tangent plane to the stoichiometric surface where the direction normal to $\Sigma_{\text {st }}$ is defined by

$$
\mathbf{n}=\left.\frac{\nabla Z}{|\nabla Z|}\right|_{\Sigma_{\mathrm{st}}} .
$$

The evolution in space and time of the FHB is then governed by the velocity of incoming gases $\mathbf{u}$ projected on the FHB, $\mathbf{u} \cdot \mathbf{m}$, and the displacement speed of the FHB relative to the flow, which is modeled in flame hole dynamics as an edge flame with velocity $V_{e}(\chi)$, according to

$$
\frac{d \mathbf{r}_{i}}{d t}=\left[\mathbf{u} \cdot \mathbf{m}-V_{e}(\chi)\right] \mathbf{m},
$$




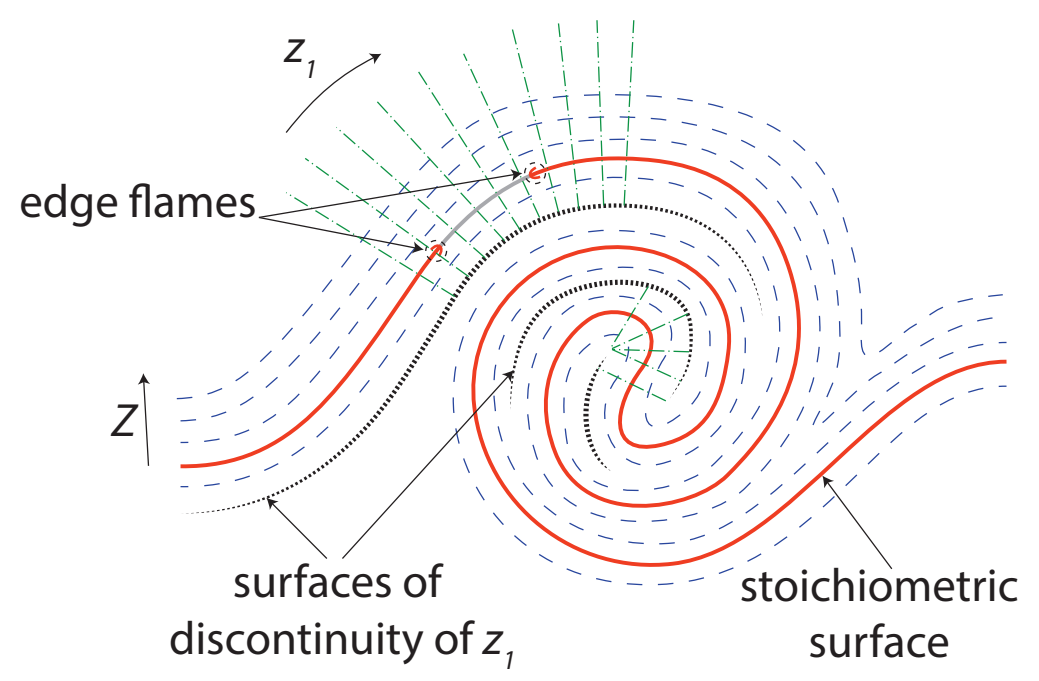

Figure 2: Sketch of the coordinate transformation used in the flame hole dynamics formulation. The surfaces of discontinuity (indicated as dotted lines in black) are manifolds where the extension in the orthogonal coordinate becomes multi-valued (although this happens away from the flame, in general).

see [28]. Therefore, the expansion or contraction of the flame hole involves two components: the flow velocity that can advect the FHB and the intrinsic edge-flame speed that couples growth or shrinkage of the flame hole depending on the magnitude of $\chi$. So far, the physical process of FHB propagation has been described in as general terms as possible with the only assumption that $V_{e}(\chi)$ exists. Further explicit closure for this quantity is required to implement this framework concretely. $V_{e}(\chi)$ is normally obtained from the solution of a two-dimensional edge flame eigenvalue problem where all details of chemistry and transport are accurately resolved. This calculation is done offline before the coupled flow and flame hole dynamics simulation. Several research groups have made progress in solving this difficult nonlinear eigenvalue problem $[32,33,34,35]$, and it is assumed that a user will possess of the correct $V_{e}(\chi)$ for the reactive mixture and thermodynamic conditions of the flame being simulated.

As it can be imagined, the modeling of flame holes using Eq. (8) is exceedingly complicated since it requires tracking the evolution and the generation of new holes throughout space in a proper curvilinear coordinate system attached to the stoichiometric surface. The solution can be best represented in a special orthogonal curvilinear coordinate system constructed by first adopting a coordinate along the normal to the $Z$ isosurfaces, as in one-dimensional flamelets, and a tangent coordinate system to those surfaces, denoted by $\mathbf{z}=\left(z_{1}, z_{2}\right)$ (which for the time being does not need to be precisely defined). Figure 2 shows a sketch of the situation on a non-trivial stoichiometric surface. In these coordinates, Eq. (8) is defined exclusively along the $\mathbf{z}$ coordinate. Note that the explicit construction of the global coordinate transformation $\mathbf{x} \mapsto(Z, \mathbf{z})$ is very difficult; there are even surfaces of discontinuities generated from different sections of the curved stoichiometric surface (but this is not a problem from the flame modeling perspective because these surfaces of discontinuity are generally far from the flame). But, one can observe that the explicit generation of $\mathbf{z}$ is unnecessary if we define a flame state field $\varphi(\mathbf{z}, t)$ that denotes the extent of reactivity at a 
particular point on the stoichiometric surface. $\varphi$ takes values between zero and one, according to

$$
\varphi= \begin{cases}\rightarrow 1 & \text { burning } \\ \frac{1}{2} & \text { at } \mathbf{x}=\mathbf{r}_{i} \\ \rightarrow 0 & \text { quenched }\end{cases}
$$

The other aspect of flame hole dynamics that needs modeling is the formation of quenched regions; which reside in the vicinity of $\chi(\mathbf{x}, t)>\chi_{\mathrm{q}}$. The extent of the extinguished regions depend on the degree by which $\chi$ exceeds $\chi_{\mathrm{q}}$, and it is generally an unsteady process [36, 37]. Immediately after chemical extinction, there are residual processes that take place and lead to the actual quenching of the flame. During this phase, after the temperature has been reduced below the cross-over extinction temperature, complete extinction is regulated by diffusion of the remaining thermal energy from the flame hole region [36]. In terms of $\varphi$, whose equation will be defined more precisely in Section 3.3, extinction can be incorporated by introducing a sink in the governing equation for $\varphi$, of the form

$$
\left[\frac{D \varphi}{D t}\right]_{s}=-\dot{Q}(\varphi, \chi)
$$

where $[D / D t]_{s}$ is a semi-Lagrangian derivative following the flame surface, which will be described in the next section, and $\dot{Q}$ is a sink law that accounts for extinction and needs to be provided; see Appendix A.2. If $\chi\left(\mathbf{x}_{s}, t\right)$ of a flame surface element with position vector $\mathbf{x}_{s}$ exceeds $\chi_{\mathrm{q}}$, then the sink $\dot{Q}$ will start to quench that region of the flame, i.e., $\varphi\left(\mathbf{x}_{s}, t\right) \rightarrow 0$. The time scale over which quenching takes place is denoted by $\tau_{\mathrm{q}}$. In a more precise analysis, extinction can be defined (approximately) in terms of the more physically realistic flame impulse [37]. The criteria establishes that the flame will quench if $\chi$ exceeds $\chi_{\mathrm{q}}$ for a sufficiently large amount of time (precisely defined through an integral); this requires a slightly more general form of the sink in Eq. (10) but it can be accommodated without difficulties.

The concepts described above involve a Lagrangian-like dynamic phenomena, Eq. (8), with an Eulerian-like process, Eq. (10). Furthermore, attempting to solve Eq. (8) embedded in a turbulent flow environment quickly becomes impractical due to the bookkeeping complexity of evolution, creation and destruction of FHB. Therefore, it is desirable to recast Eq. (8) in a purely Eulerian framework that is compatible with Eq. (10) and, more importantly, that can be implemented alongside the hydrodynamics. The main mathematical difficulty here is that $\varphi$ is really defined on a moving (and usually multiply connected) surface that is defined implicitly and whose total area is not constant. The re-formulation of the flame hole dynamics method allows for a modeled (finite) FHB propagation speed as well as taking into account the effect of the flow and extinction through the use of a level-set-like field defined on a moving curvilinear surface. This flame state field, $\varphi$, obeys a PDE describing the flame hole evolution, as described next. The formulation of this PDE and the efficient numerical implementation of such framework are the main goals of the paper.

\section{Evolution equations on general surfaces}

Let us consider a scalar field $\varphi_{s}$ that obeys an evolution equation of the form

$$
\left[\frac{D \varphi_{s}}{D t}\right]_{s}=\frac{\partial \varphi_{s}}{\partial t}+\mathbf{u}_{s} \cdot \nabla_{s} \varphi_{s}=0
$$


where all quantities are defined on an arbitrary surface $\Sigma$ (in this section the subscript "st" is not used since we discuss properties valid on arbitrary surfaces), $\mathbf{u}_{s}$ is a vector defined on the tangent space of $\Sigma$, denoted by $T_{\Sigma}$. The differential operator $\nabla_{s}$ represents the proper surface gradient on $\Sigma$. The surface gradient can be defined as the orthogonal projection of the Cartesian gradient onto $T_{S}, \nabla_{s}=P \nabla$, where $P$ is a projection operator to be defined shortly $[1,5,38,39]$; also, it can be defined in terms of the directional derivative on the surface $[40,41]$ or the intrinsic coordinate system [42]. Following Simon [40], let $\Sigma$ be at least a $\mathcal{C}^{1}$ two-dimensional submanifold of $\mathbb{R}^{3}$. Let us introduce the embedding defined by the intrinsic coordinate system $\mathbf{z} \in U \subset \mathbb{R}^{2}, \mathbf{z} \mapsto \mathbf{x} \in \Sigma$ and assume that $\varphi_{s}(\mathbf{z}, t)$ is a $\mathcal{C}^{1} \times \mathcal{C}^{1}$ function, where the first coordinate denotes the spatial coordinates of the surface and the second coordinate denotes time. By definition, $\varphi_{s}$ is a $\mathcal{C}^{1}$ function on $\mathbf{z}$ only if it is the restriction to $\Sigma$ of a $\mathcal{C}^{1}$ function $\varphi: M \times \mathbb{R} \rightarrow \mathbb{R}$, with $M$ being an open subset $M \subset \mathbb{R}^{3}$ such that $\Sigma \subset M . \varphi_{s}$ is related to $\varphi$ throughout

$$
\varphi_{s}(\mathbf{z}, t)=\varphi\left(\mathbf{x}_{s}, t\right)
$$

with $\mathbf{x}_{s}=\mathbf{x}(\mathbf{z}, t)$ denoting the position vector in the embedding space. Here, it is not assumed that a single coordinate system $\mathbf{z}$ is uniformly valid over all $\Sigma$. Instead, as it is done customarily, it is presumed that a set of differentiable coordinate systems or mappings, $\mathbf{z}_{k}$, exist on overlapping subsets $U_{k}$ of $\Sigma$; these local charts $\left(\mathbf{z}_{k}, U_{k}\right)$ cover all $\Sigma$ through an atlas [43]. For notationally simplicity, we denote the coordinate system as $\mathbf{z}$, implying that the corresponding $\mathbf{z}_{k}$ on the appropriate $U_{k}$ is being used locally.

\subsection{Spatial considerations}

Let $\mathbf{b}_{\alpha}$, with $\alpha=1,2$, denote the covariant base vectors of the surface, where

$$
\mathbf{b}_{\alpha}=\frac{\partial \mathbf{x}}{\partial z^{\alpha}}
$$

and the normalized counterparts

$$
\mathbf{t}_{\alpha}=\frac{\mathbf{b}_{\alpha}}{\left|\mathbf{b}_{\alpha}\right|}
$$

First, we wish to define an orthogonal projection tensor $P: \mathbb{R}^{3} \rightarrow T_{S}$, i.e., that takes an arbitrary vector in $\mathbb{R}^{3}$ and projects it to $T_{S}$ (still defined in the coordinates of $\mathbb{R}^{3}$ ). Strictly speaking $P$ is defined at each point $\mathbf{x}$ and a notation such as $P_{\mathbf{x}}$ would be more explicit, but unless it is required to avoid ambiguity we will not denote the explicit dependence of $P$ on $\mathbf{x}$. The orthogonal projection tensor has the properties that $P=P^{\top}, P=P^{2}$ and that it transforms every vector in $T_{S}$ into itself [42]. Consider now an arbitrary vector $\mathbf{v}$ at a point $\mathbf{x}$ in $\mathbb{R}^{3}$ and split the vector, according to

$$
\mathbf{v}=\mathbf{v}_{T}+v_{n} \mathbf{n},
$$

where $\mathbf{n}$ is the surface normal (Eq. (7)) and $v_{n}$ and $\mathbf{v}_{T}$ denote the normal and tangential components of $\mathbf{v}$ to the surface, respectively. Eq. (15) can also be written using $\mathbf{v}_{T}=P \mathbf{v}$ as

$$
\mathbf{v}=(P+\mathbf{n} \otimes \mathbf{n}) \mathbf{v}
$$

Rearranging terms in Eq. (16) and noting that $\mathbf{v}$ is arbitrary leads to

$$
P=I-\mathbf{n} \otimes \mathbf{n} .
$$


One can verify that this projection has the required properties $P=P^{\top}, P=P^{2}$, and so is an orthogonal projection of a Cartesian vector into $T_{S}$.

Now, the surface gradient will be defined using the method that employs the directional derivative on the surface in the intrinsic coordinates of $\Sigma$ [42]. The gradient operator is a linear transformation that given a function $\varphi_{s}$ and a particular direction, say an arbitrary tangent space vector $\mathbf{a}=\left(a^{1}, a^{2}\right)$, defines the derivative of $\varphi_{s}$ along such direction by

$$
\mathbf{a} \cdot \nabla_{s} \varphi_{s}(\mathbf{z})=P \nabla \varphi(\mathbf{x}),
$$

In terms of the smoothly extended $\varphi$, Eq. (12), the definition of the surface gradient for the scalar $\varphi_{s}$ can be extended to $\varphi$ at a point $\mathbf{x}$ on the surface, according to

$$
\nabla_{s} \varphi_{s}(\mathbf{z})=\frac{\partial \varphi_{s}}{\partial z^{\beta}} \mathbf{b}^{\beta}=\frac{\partial \varphi}{\partial \mathbf{x}} \cdot \frac{\partial \mathbf{x}}{\partial z^{\beta}} \mathbf{b}^{\beta}=\left(\frac{\partial \varphi}{\partial \mathbf{x}} \cdot \mathbf{b}_{\beta}\right) \mathbf{b}^{\beta}=P \nabla \varphi(\mathbf{x}),
$$

since the scalar product with $\mathbf{b}_{\beta}$ will take only the part of $\nabla \varphi$ that projects along the covariant direction vectors, and these will then be expressed in the corresponding basis function of $\mathbb{R}^{3}$, which is the same as the action of $P$.

\subsection{Moving surface considerations}

In our case, the surface defined in terms of an embedding function, $Z(\mathbf{x}, t)$, according to Eq. (3), depends on time. Now, the projection of the regular gradient of $\varphi$ on $T_{S}$ can be simplified if $\varphi$ has the following property:

$$
\nabla Z \cdot \nabla \varphi=0, \quad \forall \mathbf{x}_{s}
$$

since this implies

$$
P \nabla \varphi=\nabla \varphi .
$$

We denote by $E(t)$ the extension operator that enforces Eq. (20), or equivalently

$$
\varphi(\mathbf{x}, t)=E(t) \varphi_{s}(\mathbf{z}, t)=E(t) \varphi\left(\mathbf{x}_{s}, t\right) .
$$

Note that the extension is nonlocal: at each instant of time, the extension affects the entire domain. For this reason, the spatial dependence is omitted. With these new definitions, we can rewrite Eq. (11) according to

$$
\frac{\partial \varphi_{s}}{\partial t}+\mathbf{u}_{s} \cdot \nabla_{s} \varphi_{s}=\frac{\partial \varphi_{s}}{\partial t}+\mathbf{u}_{s} \cdot \nabla \varphi=0
$$

and the time-derivative term can be expanded using Eq. (12) to

$$
\frac{\partial \varphi_{s}}{\partial t}=\frac{\partial \varphi}{\partial t}+\nabla \varphi \cdot \frac{\partial \mathbf{x}_{s}}{\partial t}
$$

giving

$$
\frac{\partial \varphi}{\partial t}+\left(\mathbf{u}_{s}+\frac{\partial \mathbf{x}_{s}}{\partial t}\right) \cdot \nabla \varphi=0 .
$$

It is important to note that the solution of Eq. (25) is not guaranteed to match exactly the solution of Eq. (11), on $\Sigma$ at all times; even if $\varphi$ satisfies Eq. (22) at $t=0$. To ensure consistency, one would require appending Eq. (22) to Eq. (25). This can be accomplished computationally in several ways. One strategy is to employ a fractional step method where an intermediate $\varphi$ obtained from Eq. (25) 
applied to the whole three-dimensional domain is followed by enforcement of Eq. (22). Another approach is to fuse Eq. (22) and Eq. (25) using a penalty term, according to

$$
\frac{\partial \varphi}{\partial t}+\left(\mathbf{u}_{s}+\frac{\partial \mathbf{x}_{s}}{\partial t}\right) \cdot \nabla \varphi=\lambda\left[E(t) \varphi\left(\mathbf{x}_{s}, t\right)-\varphi(\mathbf{x}, t)\right]
$$

where $\lambda$ is a strength parameter that controls how close is the solution of Eq. (26) to Eq. (22). This can now be integrated in time using a semi-implicit time integration method, although this has only been demonstrated with stationary surfaces, see [44, 45].

To close Eq. (25) one needs the surface speed, $\frac{\partial \mathbf{x}_{s}}{\partial t}$, which is defined implicitly by Eq. (3). One feature of implicitly defined surfaces is that one cannot define a unique velocity at each point of the surface. It is possible, nevertheless, to assign a vector magnitude for a given preselected direction [46]. Assume a surface point moves in direction $\mathbf{d}$ (assumed normalized), then by definition

$$
\frac{\partial \mathbf{x}_{s}}{\partial t}=\mathbf{d}\left|\frac{\partial \mathbf{x}_{s}}{\partial t}\right|
$$

By differentiating Eq. (3) with respect to time we have

$$
\frac{\partial Z}{\partial t}+\nabla Z \cdot \frac{\partial \mathbf{x}_{s}}{\partial t}=0 \Rightarrow\left|\frac{\partial \mathbf{x}_{s}}{\partial t}\right|=-\frac{\frac{\partial Z}{\partial t}}{\mathbf{d} \cdot \nabla Z}
$$

where $\frac{\partial Z}{\partial t}$ must be determined from the governing equation for $Z$ evaluated at $\Sigma_{\text {st }}$. Now, since the velocity of the FHB is already decomposed into a component along $\Sigma_{\text {st }}$, Eq. (8), the only meaningful choice for $\mathbf{d}$ is $\mathbf{n}$; note that $\varphi$ does not change in that direction. This is consistent with the value of $\varphi$ being dragged to a new location simply due to the deformation of the surface. Introducing $\mathbf{d}=\mathbf{n}$ in Eq. (28), one can simplify Eq. (25) to

$$
\frac{\partial \varphi}{\partial t}+\left(\mathbf{u}_{s}-\frac{1}{|\nabla Z|} \frac{\partial Z}{\partial t} \mathbf{n}\right) \cdot \nabla \varphi=0
$$

which can be further simplified by account of Eq. (20) into the final equation

$$
\frac{\partial \varphi}{\partial t}+\mathbf{u}_{s} \cdot \nabla \varphi=0
$$

Now, the surface equation is expressed completely in terms of the extended $\varphi$ in the usual threedimensional space and its gradients and it can be solved using standard numerical techniques. Note that Eq. (22) still needs to be imposed. The solution of Eq. (30) together with Eq. (22) satisfies the surface evolution equation everywhere on $\Sigma$. The main achievement here is that the surface evolution problem, Eq. (11), has been replaced by a three-dimensional problem in the usual Cartesian space and it does not require a parameterization and discretization of the surface $\Sigma$. The implementation details of the extension operator, Eq. (22), are further discussed in Section 4.2.

\subsection{Transformation of the kinematic flame hole dynamics formulation}

Using the final result of the previous section, Eq. (30), it is possible to re-cast the Lagrangian formulation, Eq. (8), describing the evolution of FHB into a purely Eulerian formulation in the fixed Cartesian space. The kinematic equation for $\varphi$ is obtained from Eq. (8), by observing that 
the characteristic curves of Eq. (30) must equal $d \mathbf{r}_{i} / d t$, if the level set $\varphi=1 / 2$ is to move according to Eq. (8). Now, combining this observation with the FHB normal relationship to $\varphi$, given by

$$
\frac{\nabla \varphi}{|\nabla \varphi|}=\mathbf{m}
$$

according to the earlier convention of $\mathbf{m}$ pointing into the burning region, and incorporating Eq. (10), we have

$$
\mathbf{u}_{s}=(\mathbf{u} \cdot \mathbf{m}) \mathbf{m}-V_{e}(\chi) \mathbf{m},
$$

which can be simplified using $\nabla \varphi \cdot \nabla \varphi=|\nabla \varphi|^{2}$, leading to the master flame hole dynamics Eulerian equation

$$
\frac{\partial \varphi}{\partial t}+\mathbf{u} \cdot \nabla \varphi=V_{e}(\chi)|\nabla \varphi|-\dot{Q}(\varphi, \chi)
$$

A sink term, $\dot{Q}(\varphi, \chi)$, has been added to the right-hand side of the equation to account for extinction according to Eq. (10). The form of the source term used in later demonstrations of the algorithms is $\dot{Q}(\varphi, \chi) \propto \sqrt{\varphi}$ such that $\varphi$ decays quadratically in time in regions of scalar dissipation greater than the extinction value, see Appendix A.2.

Note that, to be precise, one must understand the right-hand side of Eq. (33) as $E(t)\left(V_{e}|\nabla \varphi|-\dot{Q}\right)$ because $\chi$ can (and generally does) vary along $\mathbf{n}$. Computationally, this projection is unnecessary when using a fractional-step approach because Eq. (22) is always enforced by reinitializing the extended field at every time step.

The governing equation, Eq. (33), is a level set equation defined on a moving surface. In this work, the $\varphi=1 / 2$ level set is used to separate between quenched and burning regions of the flame surface on which Eq. (33) is defined. Similar equations used in interfacial dynamics (two-phase flow) are different from Eq. (33) because the flame state field is not a physically conserved quantity; in interfacial dynamics, additional terms appear due to the requirements of conservation of surfactant mass [47].

Solving Eq. (33) while enforcing Eq. (22) in an efficient manner requires a careful numerical strategy to resolve well all the physical processes that this equation describes: flame hole formation, propagation and advection on the moving $\Sigma_{\text {st }}$. It is the main contribution of this paper.

\subsection{Initial conditions}

The flame hole dynamics concept assumes one possesses a known initial condition for $\varphi$. If the initial condition is not known precisely, but one knows that there is extinction taking place in the flame, the simplest choice is to initialize $\varphi$ to 1 and let the extinction model produce holes in the flame. If one is interested in modeling the unsteady ignition of a flame, say by forced energy deposition in some region of a cold flow mixture, the simplest method is to create a spot of $\varphi=1$ in a region that can sustain combustion (not in a region where $\chi>\chi_{q}$, of course). Flame hole dynamics is not designed in of itself to model autoignition. This phenomenon has to be incorporated separately, e.g., by temporarily reinterpreting the flame state variable as a progress variable and using an appropriate ignition model. It is similar to the situation of flamelets in nonpremixed combustion or the G-equation in premixed combustion where the creation of the flame itself is not the responsibility of those models. 


\section{Numerical scheme}

The numerical implementation of the equations describing flame hole dynamics utilizes several methods. The advection-like term in Eq. (33) is split into a divergence-like and a dilatational-like term which are treated with two different numerical methods: a conservative finite volume scheme is applied to the divergence-like term and a modified Gaussian quadrature integration is applied to the dilatational term (Section 4.1.3). A high-order WENO finite-volume formulation is used to discretize Eq. (33). WENO interpolation is used to evaluate the surface and volume integrals in the discretization (Section 4.1) and the discrete equations are integrated in time using a $3^{\text {rd }}$-order Runge-Kutta (RK3) scheme with a special antidiffusive term (or compression) that ensures that the thickness of $\varphi$ is not smeared over time because of numerical diffusion; see [48, 49]. The flame hole formation term (Section 2) is treated using a standard splitting method. Finally, the enforcement of Eq. (22) uses a specially designed extension algorithm, $E(t)$, described in Section 4.2.

\subsection{Discretization of the governing equation}

A finite-volume discretization is formulated by integrating the governing equation over a control volume, $I_{j}$, which is always taken to be a cuboid in our current implementation but could be generalized without much difficulty to other mesh geometries using already available techniques. In the finite-volume approach one solves for the cell-averaged variable, defined by

$$
\bar{\varphi}_{j}=\frac{1}{\mathrm{~V}_{j}} \iiint_{I_{j}} \varphi d \mathbf{x}
$$

where $\mathrm{V}_{j}$ is the volume of cell $I_{j}$. The cell-averaged form of Eq. (33) with the advection term split into a divergence and a dilatational term is then given by

$$
\frac{d \bar{\varphi}_{j}}{d t}+\frac{1}{\mathrm{~V}_{j}} \iint_{S_{j}} \varphi \mathbf{u} \cdot \mathbf{n}_{j} d S=\frac{1}{\mathrm{~V}_{j}} \iiint_{I_{j}} \varphi \nabla \cdot \mathbf{u} d \mathbf{x}+\frac{1}{\mathrm{~V}_{j}} \iiint_{I_{j}} V_{e}(\chi)|\nabla \varphi| d \mathbf{x}-\frac{1}{\mathrm{~V}_{j}} \iiint_{I_{j}} \dot{Q}(\varphi, \chi) d \mathbf{x},
$$

where $S_{j}$ denotes all the surfaces of the cell $I_{j}$ and $\mathbf{n}_{j}$ the corresponding surface normal vector. The approach used here whereby the advection term is casted into divergence and dilatational terms is by no means unique but it has been used successfully by many others in the past, see for example [50]. Another approach is to use a numerical method for non-conservation laws, in general formulated in terms of path integrals, see [51], but we found that this alternative strategy (possibly more mathematically elegant) was not strictly necessary to get good results. Therefore, we proceed as follows: the divergence-like term is approximated by a $5^{\text {th }}$-order accurate WENO scheme with 2-point Gaussian quadrature [52] while the dilatational-like term employs a specially conceived bilinear finite-volume integration scheme using a $4^{\text {th }}$-order WENO reconstruction and a modified Gaussian quadrature, described below. The volume-averaged flame state field, $\bar{\varphi}_{j}$, is advanced in time using a $3^{\text {rd }}$-order TVD Runge-Kutta method, developed by Gottlieb and Shu [53].

\subsubsection{Divergence-like advection terms}

The divergence part of the advection term is approximated following the method of Titarev and Toro [52], which is reproduced here partially because the new discretization method for the dilatational term - first term in the right-hand side of Eq. (35) - employs elements of the WENO 
formulation. The surface integral term in Eq. (35) is decomposed into integrals for each of the six faces of the finite-volume cell, according to

$$
\frac{1}{\mathrm{~V}_{j}} \iint_{S_{j}} \varphi \mathbf{u} \cdot \mathbf{n}_{j} d S=\frac{F_{i+\frac{1}{2}, j, k}-F_{i-\frac{1}{2}, j, k}}{\Delta x}+\frac{G_{i, j+\frac{1}{2}, k}-G_{i, j-\frac{1}{2}, k}}{\Delta y}+\frac{H_{i, j, k+\frac{1}{2}}-H_{i, j, k-\frac{1}{2}}}{\Delta z} .
$$

where the $x$-flux term $F_{i+\frac{1}{2}, j, k}$ denotes the surface integral

$$
F_{i+\frac{1}{2}, j, k}=\frac{1}{\Delta y \Delta z} \iint u\left(x_{i+\frac{1}{2}}, y, z\right) \varphi\left(x_{i+\frac{1}{2}}, y, z\right) d y d z,
$$

with $u$ denoting the $x$ component of the vector $\mathbf{u}$; similarly for the fluxes $G$ and $H$ along the other directions. This flux is approximated numerically through a four-point, two-dimensional Gaussian quadrature on the cell face, given by

$$
F_{i+\frac{1}{2}, j, k} \approx \sum_{\alpha=0}^{1} \sum_{\beta=0}^{1} w_{\alpha} w_{\beta} F^{*}\left(\varphi^{L}\left(x_{i+\frac{1}{2}}, y_{\alpha}, z_{\beta}\right), \varphi^{R}\left(x_{i+\frac{1}{2}}, y_{\alpha}, z_{\beta}\right) ; u^{L}\left(x_{i+\frac{1}{2}}, y_{\alpha}, z_{\beta}\right), u^{R}\left(x_{i+\frac{1}{2}}, y_{\alpha}, z_{\beta}\right)\right),
$$

where the weights of the Gaussian quadrature are denoted by $w_{\alpha}$ and $w_{\beta}$ and $y_{\alpha}$ and $z_{\beta}$ denote the Gaussian quadrature collocation points on the cell face in the $y$ and $z$ coordinates, respectively. The quantities $\varphi^{L}\left(x_{i+1 / 2}, y_{\alpha}, z_{\beta}\right)$ and $\varphi^{R}\left(x_{i+\frac{1}{2}}, y_{\alpha}, z_{\beta}\right)$ denote the 'left' and 'right' extrapolations to the cell face located at $x_{i+1 / 2}$, respectively. The extrapolations for $\varphi^{L}$ and $\varphi^{R}$ are obtained through WENO reconstruction whereas those of $u^{L}$ and $u^{R}$ use WENO interpolation; see Appendix B.3. The numerical flux, $F^{*}$, is obtained from the multistage upwind method (MUSTA), an iterative approximate Riemann solver that is discussed in greater detail in Appendix B.1 [52, 54, 55, 56]; see Section 5 for test cases involving the WENO-MUSTA method.

\subsubsection{Propagation and source terms}

The discretization for the FHB propagation term, $\mathcal{N}=V_{e}(\chi)|\nabla \varphi|$, is handled by a HamiltonJacobi WENO procedure [57]. We approximate the edge term by evaluating a volume integral through a Gauss quadrature,

$$
\frac{1}{V_{j}} \iiint_{I_{j}} \mathcal{N} d \mathbf{x}=\sum_{\alpha=0}^{1} \sum_{\beta=0}^{1} \sum_{\gamma=0}^{1} w_{\alpha} w_{\beta} w_{\gamma} \mathcal{N}^{\text {num }}\left(x_{\alpha}, y_{\beta}, z_{\gamma}\right)
$$

where

$$
\mathcal{N}^{\text {num }}\left(x_{\alpha}, y_{\beta}, z_{\gamma}\right)=V_{e}\left(\chi_{\alpha \beta \gamma}\right) \sqrt{\left(\varphi_{x}^{\text {up }}\right)_{\alpha \beta \gamma}^{2}+\left(\varphi_{y}^{\text {up }}\right)_{\alpha \beta \gamma}^{2}+\left(\varphi_{z}^{\text {up }}\right)_{\alpha \beta \gamma}^{2}} .
$$

Right- and left-sided approximations to the derivative $\left(\varphi_{x}^{+}\right.$and $\varphi_{x}^{-}$respectively) are calculated for use in an upwinding procedure to numerically estimate $\varphi_{x}^{\text {up }}$. We use a fourth-order, dimensionally split WENO reconstruction to evaluate the derivative (see Appendix B.3) at the Gauss quadrature points. In the $x$-direction, for example, a stencil based around $x_{i, j, k}$ is used to calculate the rightsided approximation of the derivative at $\varphi_{x}^{+}\left(x_{i}+\Delta x \alpha_{1}, y_{\beta}, z_{\gamma}\right)$ ( where $x_{i}+\Delta x \alpha_{1}$ is the $x$-coordinate of a Gauss quadrature point for the cell $I_{j}$ ) and a stencil based around $x_{i+1, j, k}$ is used to calculate the left-sided approximation, $\varphi_{x}^{-}\left(x_{i+1}-\Delta x\left(1-\alpha_{1}\right), y_{\beta}, z_{\gamma}\right)$. The values of scalar dissipation are also evaluated at the Gauss quadrature points through WENO interpolation. 
An upwinding procedure, described in Fedkiw et al. [57], is then applied to the left- and rightsided approximations at the Gauss quadrature points. If $V_{e}(\chi) \varphi_{x}^{+} \geq 0$ and $V_{e}(\chi) \varphi_{x}^{-} \geq 0$, then $\varphi_{x}^{\mathrm{up}}=\varphi_{x}^{-}$is chosen. If $V_{e}(\chi) \varphi_{x}^{+} \leq 0$ and $V_{e}(\chi) \varphi_{x}^{-} \leq 0$, then $\varphi_{x}^{\mathrm{up}}=\varphi_{x}^{+}$. If $V_{e}(\chi) \varphi_{x}^{+} \geq 0$ and $V_{e}(\chi) \varphi_{x}^{-} \leq 0$, then $\varphi_{x}^{\text {up }}=0$. Finally, if $V_{e}(\chi) \varphi_{x}^{+} \leq 0$ and $V_{e}(\chi) \varphi_{x}^{-} \geq 0$, then an intermediary variable is calculated,

$$
s=\frac{\left|\varphi_{x}^{+}\right|-\left|\varphi_{x}^{-}\right|}{\varphi_{x}^{+}-\varphi_{x}^{-}}
$$

and if $s>0$, then $\varphi_{x}^{\text {up }}=\varphi_{x}^{+}$; otherwise, $\varphi_{x}^{\text {up }}=\varphi_{x}^{-}$. The upwind derivatives are then used in Eq. (39) to calculate the FHB propagation term's contribution to the right-hand side of Eq. (33).

The algebraic source term, Eq. (A.2), is computed directly at the Gauss quadrature points, according to

$$
\frac{1}{V_{j}} \iiint_{I_{j}} \dot{Q} d \mathbf{x}=\sum_{\alpha=0}^{1} \sum_{\beta=0}^{1} \sum_{\gamma=0}^{1} w_{\alpha} w_{\beta} w_{\gamma} \dot{Q}\left(\varphi\left(x_{\alpha}, y_{\beta}, z_{\gamma}\right), \chi\left(x_{\alpha}, y_{\beta}, z_{\gamma}\right)\right),
$$

where $\varphi\left(x_{\alpha}, y_{\beta}, z_{\gamma}\right)$ and $\chi\left(x_{\alpha}, y_{\beta}, z_{\gamma}\right)$ are obtained through WENO reconstruction. The flame hole formation term implemented in this manner produces flame holes with smooth boundaries; see Section 5.4.

\subsubsection{Discretization of the dilatational-like term}

There are several strategies to integrate the dilatational-like term. One approach is described by Xing and Shu [58] for the case of the shallow water equations using a WENO scheme. Other WENO-based three-dimensional nonconservative finite-volume methods include the approach of Dumbser et al. [59,60], which use a $P_{N} P_{M}$ method in concert with a Roe-type or modified FORCE flux approximation [61]. The present approach is to use a modified Gauss quadrature rule to directly evaluate the integrals appearing in the first-term of the right-hand side of Eq. (35). The functions in this new modified Gauss quadrature are interpolated or reconstructed by the WENO method. The basic idea is to use a specific quadrature for integrals of the form

$$
\int_{-1}^{1} \varphi(x) \frac{d u}{d x}(x) d x
$$

We follow the classical approach [62] and approximate the function $\varphi$ in a Hermite polynomial, according to

$$
\varphi(x) \approx \tilde{\varphi}(x)=\sum_{\alpha=1}^{m} h_{\alpha}(x) \varphi\left(x_{\alpha}\right)+\sum_{\alpha=1}^{m} \bar{h}_{\alpha}(x) \varphi^{\prime}\left(x_{\alpha}\right),
$$

where prime denote the derivative with respect to $x$, and

$$
h_{\alpha}(x)=\left[1-2 l_{j}^{m \prime}\left(x_{\alpha}\right)\left(x-x_{\alpha}\right)\right]\left[l_{\alpha}^{m}(x)\right]^{2}, \quad \bar{h}_{\alpha}=\left(x-x_{\alpha}\right)\left[l_{\alpha}^{m}(x)\right]^{2},
$$

where

$$
l_{\alpha}^{m}(x)=\frac{\left(x-x_{1}\right) \cdots\left(x-x_{\alpha-1}\right)\left(x-x_{\alpha+1}\right) \cdots\left(x-x_{m}\right)}{\left(x_{\alpha}-x_{1}\right) \cdots\left(x_{\alpha}-x_{\alpha-1}\right)\left(x_{\alpha}-x_{\alpha+1}\right) \cdots\left(x_{\alpha}-x_{m}\right)},
$$


and $x_{\alpha}$ denote the interpolation points, yet to be determined. The function $u$ is expanded in the same manner but with a degree $m+1$ polynomial, since we need to take the derivative of the interpolating polynomial, given by

$$
\tilde{u}(x)=\sum_{b=1}^{m+1} g_{\beta}(x) u\left(x_{\beta}\right)+\sum_{b=1}^{m+1} \bar{g}_{\beta}(x) u^{\prime}\left(x_{\beta}\right) .
$$

Here, $g_{\beta}$ and $\bar{g}_{\beta}$ are analogous to $h_{\alpha}$ and $\bar{h}_{\alpha}$ from Eq. (45)-(46) except that their interpolating polynomials have degree $m+1$. The modified Gaussian quadrature seeks to estimate $\varphi(x) u^{\prime}(x)$ by multiplying their Hermite polynomials together and integrating. The derivative of $u$ is given by

$$
\tilde{u}^{\prime}(x)=\sum_{b=1}^{m+1} g_{\beta}^{\prime}(x) u\left(x_{\beta}\right)+\sum_{b=1}^{m+1} \bar{g}_{\beta}^{\prime}(x) u^{\prime}\left(x_{\beta}\right)
$$

with

$$
\begin{aligned}
& g_{\beta}^{\prime}(x)=\left[-2 l_{\beta}^{m+1}\left(x_{\beta}\right)\right]\left[l_{\beta}^{m+1}(x)\right]^{2}+\left[1-2 l_{\beta}^{m+1 \prime}\left(x_{\beta}\right)\left(x-x_{\beta}\right)\right]\left[2 l_{\beta}^{m+1}(x) l_{\beta}^{m+1 \prime}(x)\right], \\
& \bar{g}_{\beta}^{\prime}(x)=2\left(x-x_{\beta}\right) l_{\beta}^{m+1}(x) l_{\beta}^{m+1 \prime}(x)+l_{\beta}^{m+1}(x)^{2} .
\end{aligned}
$$

The combined Hermite polynomial expansion for the integrand of Eq. (43) is

$$
\begin{aligned}
\tilde{u}^{\prime}(x) \tilde{\varphi}(x)=\sum_{\alpha=1}^{m} \sum_{b=1}^{m+1}( & h_{\alpha}(x) g_{\beta}^{\prime}(x) \varphi\left(x_{\alpha}\right) u\left(x_{\beta}\right)+h_{\alpha}(x) \bar{g}_{\beta}^{\prime}(x) \varphi\left(x_{\alpha}\right) u^{\prime}\left(x_{\beta}\right) \\
& \left.+\bar{h}_{\alpha}(x) g_{\beta}^{\prime}(x) \varphi^{\prime}\left(x_{\alpha}\right) u\left(x_{\beta}\right)+\bar{h}_{\alpha}(x) \bar{g}_{\beta}^{\prime}(x) \varphi^{\prime}\left(x_{\alpha}\right) u^{\prime}\left(x_{\beta}\right)\right) .
\end{aligned}
$$

The integral of Eq. (51) is given by

$\int_{-1}^{1} \tilde{u}^{\prime}(x) \tilde{\varphi}(x) d x=\sum_{\alpha=1}^{m} \sum_{b=1}^{m+1}\left[I_{\alpha \beta}^{0} \varphi\left(x_{\alpha}\right) u\left(x_{\beta}\right)+I_{\alpha \beta}^{1} \varphi\left(x_{\alpha}\right) u^{\prime}\left(x_{\beta}\right)+I_{\alpha \beta}^{2} \varphi^{\prime}\left(x_{\alpha}\right) u\left(x_{\beta}\right)+I_{\alpha \beta}^{3} \varphi^{\prime}\left(x_{\alpha}\right) u^{\prime}\left(x_{\beta}\right)\right]$,

with

$$
\begin{array}{ll}
I_{\alpha \beta}^{0}=\int_{-1}^{1} h_{\alpha}(x) g_{\beta}^{\prime}(x) d x, & I_{\alpha \beta}^{1}=\int_{-1}^{1} h_{\alpha}(x) \bar{g}_{\beta}^{\prime}(x) d x, \\
I_{\alpha \beta}^{2}=\int_{-1}^{1} \bar{h}_{\alpha}(x) g_{\beta}^{\prime}(x) d x, & I_{\alpha \beta}^{3}=\int_{-1}^{1} \bar{h}_{\alpha}(x) \bar{g}_{\beta}^{\prime}(x) d x .
\end{array}
$$

In order for the quadrature to not involve the derivatives of either $u$ or $\varphi$ (which are unknown), $I_{\alpha \beta}^{1}, I_{\alpha \beta}^{2}$, and $I_{\alpha \beta}^{3}$ must be zero for all $a$ and $b$, a task accomplished by choosing the abscissae $x_{\alpha}$ for $\varphi$ and $x_{\beta}$ for $u$ such that the integrals vanish. The weights and abscissae can be derived by solving a nonlinear system of equations for both the values of the weights and locations of the abscissae, designed by requiring exact solutions to polynomials of a certain degree. For polynomials of combined degrees $n_{\alpha}+n_{\beta}<2 m+1$, we have

$$
\sum_{\alpha}^{n_{\alpha}} \sum_{\beta}^{n_{\beta}} w_{\alpha \beta} x_{\alpha}^{j} x_{\beta}^{k}=\int_{-1}^{1} \xi^{n_{\alpha}} \frac{d\left(\xi^{n_{\beta}}\right)}{d \xi} d \xi=\frac{\left(1+(-1)^{n_{\alpha}+n_{\beta}}\right) n_{\beta}}{n_{\alpha}+n_{\beta}} .
$$


The solution of this polynomial system of equations provides both the weights and abscissae of the modified Gaussian quadrature. We have implemented this method using both $m=2$ and $m=3$. The weights and abscissae are given in Appendix B.2.

The modified Gauss quadrature is extended to three dimensions using the regular Gaussian quadrature rule in the directions orthogonal to the derivative direction (tensor product). The values of the functions at the modified Gaussian quadrature points are estimated by WENO interpolation, e.g., evaluation for the $x$-derivative term gives

$$
\frac{1}{\mathrm{~V}_{j}} \iiint_{\mathrm{I}_{j}} \frac{\partial u}{\partial x} \varphi d \mathbf{x}=\frac{1}{\Delta x} \sum_{\alpha} \sum_{\beta} \sum_{\kappa} \sum_{\lambda} w_{l} w_{m} w_{\alpha \beta} \varphi\left(x_{\alpha}, y_{\kappa}, z_{\lambda}\right) u\left(x_{\beta}, y_{\kappa}, z_{\lambda}\right),
$$

with analogous terms in the $y$ and $z$ directions. The integration involves a significant amount of WENO interpolations and reconstructions to perform, with 48 evaluations required to evaluate the $m=3$ quadrature in one of three directions for each finite-volume cell. The method however directly evaluates the dilatational-like term using a WENO scheme consistent with the divergencelike term and since it is only applied to one scalar equation, its overall computational costs is not overwhelming; note that in the use of the whole coupled method one is also solving the Navier-Stokes equations and the extension operator described in next section.

We can estimate the numerical truncation errors of the divergence and dilatational terms generated by both the interpolation (reconstruction) error of the WENO method and the integration error of the Gaussian quadratures. Consider the surface integration of the divergence term, with a Gaussian integration of order $p$ and a WENO interpolation of order $q$. For equal grid spacing $\Delta x=\Delta y=\Delta z=\Delta$, we have

$$
\begin{aligned}
\frac{1}{\Delta^{3}} \iint f(y, z) d y d z & =\frac{1}{\Delta^{3}}\left(\iint \tilde{f}(y, z) d y d z+\iint O\left(\Delta^{q}\right) d y d z\right) \\
& =\sum_{\alpha} \sum_{\beta} w_{\alpha} w_{\beta} \tilde{f}\left(y_{\alpha}, z_{\beta}\right)+O\left(\Delta^{p-3}\right)+O\left(\Delta^{q-1}\right)
\end{aligned}
$$

where $\tilde{f}$ denotes the interpolated value of $f$. The error in one of the flux terms is order $\min (p-$ $3, q-1)$. Since these fluxes appear always in pairs, as a difference between neighboring points, one actually gains one order of accuracy above the individual flux approximations, giving a final error of $\min (p-2, q)$. A similar analysis applies to the nonconservative term, giving

$$
\begin{array}{r}
\frac{1}{\Delta^{3}} \iiint \nabla \cdot \mathbf{u}(x, y, z) \varphi(x, y, z) d x d y d z= \\
\frac{1}{\Delta^{3}} \iiint\left[\nabla \cdot \tilde{\mathbf{u}}(x, y, z)+O\left(\Delta^{q-1}\right)\right]\left[\tilde{\varphi}(x, y, z)+O\left(\Delta^{q}\right)\right] d x d y d z= \\
\frac{1}{\Delta^{3}} \iiint \nabla \cdot \tilde{\mathbf{u}}(x, y, z) \tilde{\varphi}(x, y, z) d x d y d z+O\left(\Delta^{q-1}\right)= \\
\sum \sum \sum w_{\alpha, \beta, \gamma} \nabla \cdot \tilde{\mathbf{u}}\left(x_{\alpha^{\prime}}, y_{\beta^{\prime}}, z_{\gamma^{\prime}}\right) \tilde{\varphi}\left(x_{\alpha}, y_{\beta}, z_{\gamma}\right)+O\left(\Delta^{r-3}\right)+O\left(\Delta^{q-1}\right) .
\end{array}
$$

were $r$ denotes the order of the modified Gaussian quadrature introduced above, $w_{\alpha, \beta, \gamma}$ denotes the corresponding combined quadrature weights in all directions and the primed Greek index denote the collocation points of the derivative term in the modified Gaussian quadrature. The order of accuracy for this term is therefore $\min (r-3, q-1)$. 


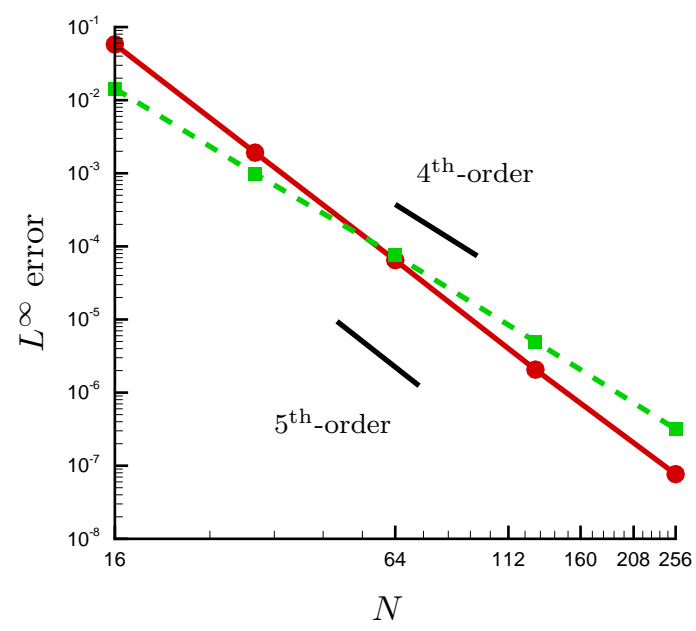

Figure 3: Scaling of the truncation error for the divergence- and dilatational-like terms for two-point quadratures The error at different resolutions is marked with square symbols for the dilatational-like term and circle symbols for the divergence-like term.

Figure 3 shows the scaling of the calculated truncation error for the whole divergence and dilatational terms using the quadrature rules described above for $u=1+a \sin (x), v=1+a \sin (y)$, $w=1+a \sin (z), \varphi=\sin (x) \sin (y) \sin (z)$ with $a=0.8, x, y, z \in[0,2 \pi]$ using grids with $16^{3}, 32^{3}, 64^{3}$, $128^{3}$, and $256^{3}$ points. For the two-point Gaussian quadrature rules we use throughout the paper, it was found that the dilatational term follows an approximately $4^{\text {th }}$-order slope with increasing resolution, and the divergence term has a $5^{\text {th }}$-order error, as expected by the previous truncation error estimates since $p=2 \times 3+1=7, r=2 \times 3+1=7$, and $q=5$.

\subsubsection{Anti-dissipative correction}

To prevent the artificial smearing of $\varphi$ on the stoichiometric surface, due to numerical dissipation, we add an antidiffusive regularization to the governing equation, Eq. (33) - which we call a compression term $[48,49]$ - based on the idea that the equilibrium solution of

$$
\frac{\partial \varphi}{\partial \tau}=\mathcal{U}_{0} \mathcal{M}(\varphi)
$$

where

$$
\mathcal{M}(\varphi)=\mathbf{n}_{\varphi} \cdot \nabla\left(\epsilon_{c}|\nabla \varphi|-\varphi(1-\varphi)\right) .
$$

with $\mathcal{U}_{0}$ large, converges to

$$
\varphi=\frac{1}{2}\left(1+\tanh \left(\frac{n}{2 \epsilon_{c}}\right)\right)
$$

with $n$ denoting the spatial coordinate normal to the $\varphi=1 / 2$ surface and $\epsilon_{c}$ is a parameter that determines the interface thickness (usually chosen proportional to the grid spacing) and the value 


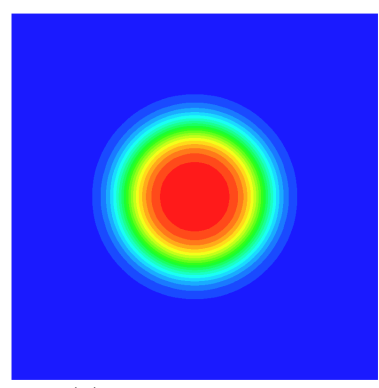

(a) Initial condition

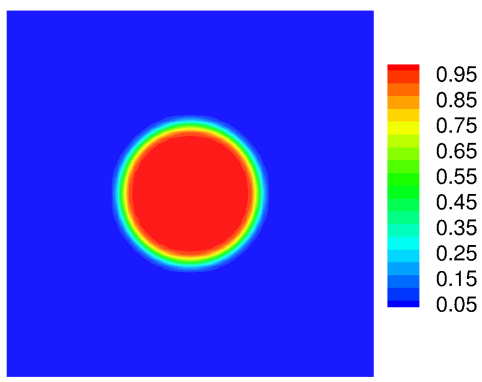

(b) After compression

Figure 4: Compression algorithm effect on a circular flame disk on a rectangular $64 \times 64 \times 2$ domain, with parameters $\mathcal{U}_{0}=1$ and $\epsilon_{c}=\max (\Delta x, \Delta y, \Delta z)=\frac{1}{63}$, and no flow velocity. A subiteration CFL number of 0.025 is used until a convergence of $\times 10^{-2}$ is obtained. The thickness of the initial profile is reduced from $8 \Delta x$ to $2 \Delta x$ through the compression. A consistent color scale from 0 to 1 is used for future images of $\varphi$.

of $\mathcal{U}_{0}$ chosen as the maximum of the norm of the velocity,

$$
\mathcal{U}_{0}=\max _{i, j, k}\left(\sqrt{u_{i, j, k}^{2}+v_{i, j, k}^{2}+w_{i, j, k}^{2}}\right) .
$$

Figure 4 shows an example of the effect of adding compression regularization to the advection of an initially diffuse cylindrical flame hole in a flat surface. The diffuse interface is compressed to a specified width (here, two grid cells wide) by adding the right-hand side of Eq. (58) to Eq. (35). Note that the initially very diffuse flame hole becomes sharp after the first time-step since Eq. (58) is allowed to take enough sub-steps to fully compress the interface. This strategy yields sharp but mesh-representable transitions between the burning and quenched states. Since the compression is acting all along the evolution of the governing equation, we find that it is typically only necessary to perform one or two compression sub-cycles at each time step to keep the FHB sharp. Furthermore, compression does not seem to affect merging or spliting of flame holes in any measurable way in all the tests we conducted.

\subsection{The extension operator}

The extension operator, $E(t)$, takes information on $\Sigma_{\text {st }}$ and propagates it smoothly into the surrounding three-dimensional domain along the direction defined by $\nabla Z$; the basic condition that must be enforced is Eq. (20). We choose a hybrid method for the extension. The extension is done in two bands: a closest point extension is applied in a band near the surface and a PDE-type extension discussed later in this section is applied outside of that band, using values from the closest point extension $[6,7,8,10]$ as the interior Dirichlet condition. This mixture of methods allows us to use a high-order extension close to the surface, where the accuracy of the surface evolution is affected, and a faster but less accurate extension where the solution does not affect the accuracy of the surface evolution.

For the closest point extension, a band is formed around the surface such that all points are within a distance of $n_{b} \max (\Delta x, \Delta y, \Delta z)=n_{b} \Delta_{\max }$ from the surface in any direction, where $n_{b}$, discussed later in this section, is some positive integer that sets the width of the band. Within this 
band, the closest point on the surface to a grid point $x_{i, j, k}$ is calculated by use of a local, quadratic approximation to $\Sigma_{\text {st }}$ as in Leung et al. [10], given by

$$
Z_{L S Q, 2}(\mathbf{x})=p+\mathbf{q}^{\top} \mathbf{x}+\frac{1}{2} \mathbf{x}^{\top} \mathbf{H} \mathbf{x}
$$

where the coefficients of the approximation $-p, \mathbf{q}$, and $\mathbf{H}$ - are obtained through a weighted least squares approximation using the neighboring points; i.e., for a point $\mathbf{x}_{j}$, a cell of neighboring points $\mathbf{x}_{j}+n(\Delta x, \Delta y, \Delta z)$, with $n$ ranging between -1 and 1 , are used to form the least squares system. The weighting, at a point $\mathbf{x}_{j}$ in the least squares system, is an approximation of the distance using the mixture fraction,

$$
\mathbf{W}_{Z, j}=\frac{\min \left(\left|Z\left(\mathbf{x}_{j}\right)-Z_{\mathrm{st}}\right|\right)}{\left|Z\left(\mathbf{x}_{j}\right)-Z_{\mathrm{st}}\right|}
$$

The distance between the grid point and the surface is then minimized by moving along the coordinate normal to the quadratic approximation of the surface,

$$
\mathbf{c p}\left(\mathbf{x}_{j}\right)=\mathbf{x}_{j}-\eta \nabla Z_{L S Q}
$$

with $\eta$ chosen such that $Z(\mathbf{c p}(\mathbf{x}))=Z_{\text {st }}$. To find $\eta$, we use a Newton iteration to solve

$$
f(\eta)=\operatorname{det}(\mathbf{I}+\eta \mathbf{H})^{2}\left(p-Z_{\mathrm{st}}\right)+\operatorname{det}(\mathbf{I}+\eta \mathbf{H}) \mathbf{q}^{\top} \mathbf{B} \zeta+\frac{1}{2}(\mathbf{B} \zeta)^{\top} \mathbf{H}(\mathbf{B} \zeta)=0,
$$

with $\zeta=\mathbf{x}_{j}-\eta \mathbf{q}$ and $\mathbf{B}=\operatorname{det}(\mathbf{I}+\eta \mathbf{H})(\mathbf{I}+\eta \mathbf{H})^{-1}$. The derivative of this function is

$$
f^{\prime}(\eta)=2 \operatorname{det}(\mathbf{I}+\eta \mathbf{H}) \operatorname{det}(\mathbf{I}+\eta \mathbf{H})^{\prime}\left(p-Z_{\mathrm{st}}\right)+\operatorname{det}(\mathbf{I}+\eta \mathbf{H}) \mathbf{q}^{\boldsymbol{\top}} \psi^{\prime}+\operatorname{det}(\mathbf{I}+\eta \mathbf{H})^{\prime} \mathbf{b}^{\boldsymbol{\top}} \psi+\psi^{\boldsymbol{\top}} \mathbf{H} \psi^{\prime},
$$

with $\psi=\mathbf{B} \zeta$ and $\psi^{\prime}=\mathbf{B}^{\prime} \zeta-\mathbf{B q}$. The value of $\eta$ is then computed iteratively to convergence by,

$$
\eta_{n+1}=\eta_{n}-\alpha_{D} \frac{f(\eta)}{f^{\prime}(\eta)}
$$

where $\alpha_{D}$ is a damping coefficient chosen to be 0.5 .

Since, for a general surface, the closest point is almost never a grid point of the computational domain, an estimate of $\varphi(\mathbf{c p}(\mathbf{x}))$ is also required. We use a weighted least squares approximation of $\varphi$ centered around the nearest point to $\mathbf{c p}(\mathbf{x})$ on the grid,

$$
\varphi_{L S Q, 2}\left(\mathbf{c p}\left(\mathbf{x}_{j}\right)\right)=\hat{p}+\hat{\mathbf{q}}^{\top} \mathbf{c p}\left(\mathbf{x}_{j}\right)+\frac{1}{2} \mathbf{c p}\left(\mathbf{x}_{j}\right)^{\top} \hat{\mathbf{H}} \mathbf{c p}\left(\mathbf{x}_{j}\right),
$$

where the hat indicates that the coefficients are calculated based on values of $\varphi$ with weighting,

$$
\mathbf{W}_{\varphi, j}=\frac{\min \left(\left|\mathbf{x}_{j}-\mathbf{c p}(\mathbf{x})\right|\right)}{\left|\mathbf{x}_{j}-\mathbf{c p}(\mathbf{x})\right|}
$$

Since we are interested in the application of the algorithm to very complicated surfaces, we find it necessary to incorporate certain regularizations or numerical entropy solution strategies to obtain a robust functioning of the method. These corrections are not often activated in a typical domain, but when they are, they enable calculation on otherwise impossible surface configurations that are not statistically significant but must be treated satisfactorily. As this approximation is not total variation diminishing, we limit value produced by this approximation to be within the range of 
values used in the least squares system. The right-hand side of flame hole dynamics equation, Eq. (33), is then computed on each point used in the least squares approximation to $\varphi(\mathbf{c p}(\mathbf{x}))$ in order to advance the solution in time. The width of the band is chosen such that the closest point extension is computed at all points used to evaluate the righthand side of Eq. (33). For cases with highly varying $Z$ fields, like the shear layer in figure 15), the Newton method Eq. (67) can sometimes fail to converge. At these points, a linear approximation to the surface is employed instead, given by

$$
Z_{L S Q, 1}(\mathbf{x})=p_{1}+\mathbf{q}_{1}^{\top} \mathbf{x}
$$

where $p_{1}$ and $\mathbf{q}_{1}$ represent the least square coefficients for the linear approximation. The closest point can be found exactly as

$$
\mathbf{c p}\left(\mathbf{x}_{j}\right)=\mathbf{x}_{j}-\left(p_{1}-Z_{\mathrm{st}}\right) \frac{\mathbf{q}_{1}}{\mathbf{q}_{\mathbf{1}}^{\top} \mathbf{q}_{\mathbf{1}}}
$$

and the resulting estimate for the closest point can be used to evaluate $\varphi_{L S Q, 2}(\mathbf{c p}(\mathbf{x}))$. We additionally test whether the linear approximation gives a reasonable solution by checking if $\left|\mathbf{c p}(\mathbf{x})-\mathbf{x}_{j}\right|>$ $n_{b} \Delta_{\max }$. If it fails this test, then value of $\varphi$ at that point is set equal to the average of neighboring points for which the closest point extension passes this test.

The width of the band, $n_{b}$, is chosen based on the width of the cell used in the least squares approximation of $\varphi$ and the width of the stencil used to solve Eq. (33). The flame hole dynamics equation is solved at every point used in the estimation of $\varphi_{L S Q, 2}\left(\mathbf{c p}\left(\mathbf{x}_{j}\right)\right)$, and the band width is chosen such that every point used has been extended through the closest point method. The stencil width is therefore the maximum distance a point in the least squares cell for $\varphi$ can be from the surface $\left(2 \Delta_{\max }\right)$ in any direction, plus the width of stencil discretizing Eq. (33) $\left(3 \Delta_{\max }\right)$, resulting in a value of $n_{b}=5$. This choice of band width is consistent with the analysis of band width for the closest point method in Macdonald and Ruuth [7].

The closest point extension, while higher order, is both expensive and inaccurate at points far away from the surface. In order to extend the field into a large band in the domain, a PDE-based extension is used. This type of extension has desirable properties $[63,64]$ for solving the extension in a very large band around the surface. They rely on solving a PDE that satisfies the extension condition Eq. (20), achieved by the steady solution of

$$
\frac{\partial \varphi}{\partial \tau}+\operatorname{sgn}\left(Z-Z_{\text {st }}\right) \mathbf{n} \cdot \nabla \varphi=0
$$

where $\tau$ is a pseudo-time variable that has only a transitory role as the equation is solved until the solution is stationary. In this method, there is no difficulty at the boundaries of the computational domain and simple outflow conditions for the scalar advection equation can be used. However, the computational effort associated with this method is significant because one needs to take many pseudo-time steps for the solution to Eq. (72) to become stationary. This can be avoided solving directly for the modified steady-state version of Eq. (72), given by

$$
\operatorname{sgn}\left(Z-Z_{\text {st }}\right) \tilde{\mathbf{n}} \cdot \nabla \varphi=\nabla \cdot(\varepsilon(\mathbf{x}) \nabla \varphi)
$$

where

$$
\tilde{\mathbf{n}}=\frac{\nabla Z}{|\nabla Z|+g_{\epsilon}},
$$

is a regularized approximation of the $\Sigma_{\text {st }}$ normal required to ensure good behavior when approaching critical points in $Z$ or the free streams (where $Z$ is constant); further discussion on this regularization 
follows below. The parameter $\varepsilon(\mathbf{x})$ is introduced to ensure stability and it scales with $\Delta_{\text {avg }}=$ $(\Delta x \Delta y \Delta z)^{1 / 3}$ so that Eq. (73) and Eq. (72) are consistent and Eq. (73) converges to the same solution under refinement. The implementation of the method is a generalization of a recent implicit extension algorithm developed in [65], modified to ensure the idempotence of the extension operator; that is $E^{2}=E$. First, a band of grid points that are within $\Delta_{\max }=\max (\Delta x, \Delta y, \Delta z)$ from the stoichiometric surface are identified as the boundary points $\varphi_{\mathrm{bc}}$ (Dirichlet boundary condition data) of Eq. (73). Second, discretization of Eq. (73) is done through a first-order upwind method for the advection term, where the $\mathrm{x}$ derivative (similarly for the $\mathrm{y}$ and $\mathrm{z}$ derivatives) is given by

$$
D_{\mathrm{up}, x} \varphi=m_{x} \frac{\varphi_{i, j, k}-\varphi_{i-m_{x}, j, k}}{\Delta x},
$$

where $m_{x}=\operatorname{sgn}\left(a_{x}\right)$, and

$$
\mathbf{a}=\left(a_{x}, a_{y}, a_{z}\right)=\operatorname{sgn}\left(Z-Z_{\text {st }}\right) \tilde{\mathbf{n}},
$$

with $\tilde{\mathbf{n}}$ given by Eq. (74) approximated by central differences. The $\mathrm{x}$ derivatives of the diffusion terms are discretized according to

$$
D_{x}\left(\varepsilon D_{x} \varphi\right)=\frac{1}{2 \Delta x^{2}}\left[\left(\varepsilon_{i+1, j, k}+\varepsilon_{i, j, k}\right)\left(\varphi_{i+1, j, k}-\varphi_{i, j, k}\right)-\left(\varepsilon_{i, j, k}+\varepsilon_{i-1, j, k}\right)\left(\varphi_{i, j, k}-\varphi_{i-1, j, k}\right)\right],
$$

which in the case of constant coefficient diffusion reduces to the standard central stencil for the second-order derivative; similarly for the other coordinate directions. After rearrangement of the variables, the extension algorithm requires the solution of

$$
\mathcal{L} \varphi=b_{\mathrm{bc}}
$$

where

$$
\mathcal{L}=a_{x} D_{\mathrm{up}, x}^{\prime}+a_{y} D_{\mathrm{up}, y}^{\prime}+a_{z} D_{\mathrm{up}, z}^{\prime}-D_{x}^{\prime} \varepsilon D_{x}^{\prime}-D_{y}^{\prime} \varepsilon D_{y}^{\prime}-D_{z}^{\prime} \varepsilon D_{z}^{\prime},
$$

denotes the advection-diffusion operator stripped of the points affecting $\varphi_{\mathrm{bc}}$ (denoted with primes) and $b_{\mathrm{bc}}$ denotes the parts of the stencils involving $\varphi_{\mathrm{bc}}$.

Figure 5 shows the result of solving Eq. (78) when discretized in the Cartesian grid for the case of a circular flame disk on a curved sinusoidal stoichiometric surface. The translucent isosurfaces show the extension of the flame state field to the full three-dimensional domain for different level sets of $\varphi$. The values of $\varphi$ on the surface are propagated along the translucent isosurfaces. The flame state field $\varphi$ is extended smoothly into the domain, perpendicular to the iso-surfaces of the mixture fraction and along the normal $\mathbf{n}$, as depicted with translucent iso-surfaces of constant $\varphi$. Note that the extended $\varphi$ iso-surfaces $(\varphi=0.25,0.5$ and 0.75$)$ concentrate as they move away from $\Sigma_{\text {st }}$ due to the curvature of this surface and the interaction with the flame state values on the other sections of this surface.

Problems with this method can occur in practical applications when there may be regions of the domain where $|\nabla Z|$ approaches zero (a case not considered in figure 5). There are two typical scenarios when this can occur: in unmixed regions of a flow such as at the edge of a shear layer or at the outer extent of a jet flow, or at critical points in a turbulent flow. Each scenario is handled differently. To ensure stability of the method for $Z$ fields containing critical points, $|\nabla Z| \approx 0$, a proper choice of $\varepsilon$, related to the regularization used in Eq. (74), must be used in Eq. (73). Our numerical experiments were successful using the following strategy. In the first phase, an indicator function, $I_{\varepsilon}$, was defined according to

$$
I_{\varepsilon}=\varepsilon_{0}\left[\Delta_{\mathrm{avg}}+\ell\left(\frac{g_{\epsilon}}{g_{\epsilon}+|\nabla Z|}\right)\right]
$$




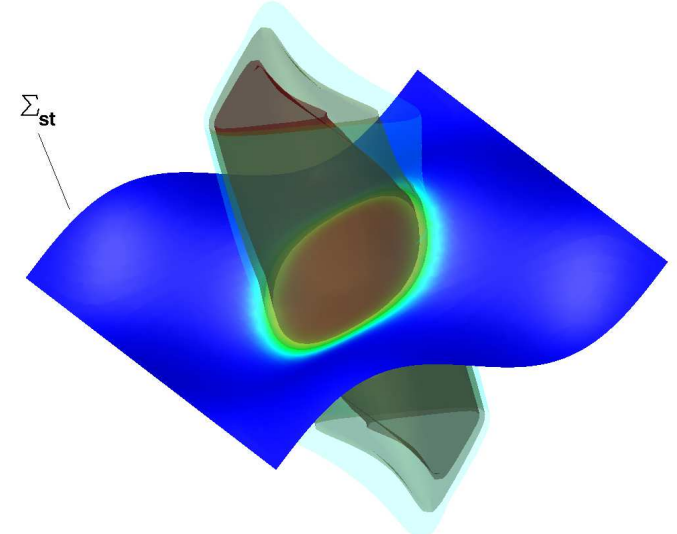

Figure 5: Example of extension of $\varphi$ defined on a sinusoidal surface using the Cartesian grid-based method, with $Z_{\mathrm{st}}=0.5$. Surface is given by $Z_{\mathrm{st}}=\operatorname{asin}\left(2 \pi / L_{x} x\right) \cos \left(2 \pi / L_{y} y\right)$, with $a=0.1 L_{x}$. Color scale used is consistent with scale in figure 4.

where $\ell$ denotes the characteristic lengthscale of variation of $\nabla Z$ near critical points and $g_{\epsilon}$ denotes the characteristic cutoff scale for good behavior of $\mathbf{n}$, a small fraction of $|\nabla Z|$ overall. The intention of Eq. (80) is to ensure that $\varepsilon$ behaves as $\Delta_{\text {avg }}$ for large values of $\nabla Z$, while it approaches $\ell$ when $\nabla Z$ is very small (it is assumed that $\Delta_{\text {avg }} \ll \ell$ if $Z$ is well-resolved by the mesh). The constant $\varepsilon_{0}$ is independent of the grid spacing or $Z$, while $g_{\epsilon}$ and $\ell$ depend on the degree of variation of $\nabla Z$ in the domain (these parameters can be selected reasonably well by an initial analysis of $Z$ throughout the domain). In the second phase, the indicator function is smoothed over several grid cells by the application of a filter of width $2 \Delta_{\text {avg }}$, giving

$$
\varepsilon(\mathbf{x})=\left(\frac{1}{8 \pi \Delta_{\text {avg }}^{2}}\right)^{3 / 2} \iiint I_{\epsilon}\left(\boldsymbol{x}-\boldsymbol{x}^{\prime}\right) \exp \left(\frac{-\left|\boldsymbol{x}-\boldsymbol{x}^{\prime}\right|^{2}}{8 \Delta_{\text {avg }}^{2}}\right) d \boldsymbol{x}^{\prime},
$$

The resulting $\varepsilon$ field is smooth and localized around critical points. This ensures the stability of the method while still limiting the effect diffusion has on $\varphi$.

The previous selection of $\varepsilon$ resolves the treatment of critical points. For unmixed regions, where $Z$ is 0 or 1 , there is no flame and the value of $\varphi$ can be set to zero safely because it does not affect the calculation of the state of the gas mixture. Therefore, the extension is only performed on regions where mixing is present: $0+Z_{\epsilon}=Z_{-}<Z<Z_{+}=1-Z_{\epsilon}$, where we have used $Z_{\epsilon}=0.001$ satisfactorily in all our simulations. A Robin boundary condition is used at this boundary, $Z=Z_{ \pm}$, to ensure stability of the extension algorithm when disconnected regions of slightly mixed fluid near the exterior boundary are present or form during a simulation (multiply connected case), a scenario that occurs in practice in turbulence simulations when detachment of mixture fraction blobs from 


\begin{tabular}{c|cccc} 
Resolution & $L^{1}$ & $L^{2}$ & $L^{\infty}$ & Order \\
\hline $16 \times 16 \times 2$ & $2.67 \times 10^{-4}$ & $1.22 \times 10^{-3}$ & $7.17 \times 10^{-3}$ & \\
$32 \times 32 \times 2$ & $4.87 \times 10^{-6}$ & $3.18 \times 10^{-5}$ & $2.96 \times 10^{-4}$ & 5.26 \\
$64 \times 64 \times 2$ & $1.05 \times 10^{-7}$ & $9.42 \times 10^{-7}$ & $1.22 \times 10^{-5}$ & 5.08 \\
$128 \times 128 \times 2$ & $3.45 \times 10^{-9}$ & $4.33 \times 10^{-8}$ & $7.42 \times 10^{-7}$ & 4.44
\end{tabular}

Table 1: Advection of a smooth, sinusoidal $\varphi$ in the $x$-direction on a flat surface. Error comparison is between the initial condition and the solution after one flow through time. Order of accuracy is estimated based on the $L^{2}$-norm.

the main turbulence region takes place. Now, the Robin boundary condition is given by

$$
\alpha_{R} \varphi+\frac{\partial \varphi}{\partial n}=0
$$

where $n$ is the normal direction to the boundary, and $\alpha_{R}$ is a small parameter that determines the relative weight of the exterior boundary data in the boundary condition; note that for $\alpha_{R}$ sufficiently small, the boundary condition $\varphi=0$ is effective only when the normal gradient is negligible (as intended). Within the domain $Z_{-}<Z<Z_{+}$, the boundary condition for the extension algorithm is an internal Dirichlet condition using values from the closest point extension. The resulting equations are solved using an algebraic multigrid method (BoomerAMG) provided by the library hypre [66]. Finally, note that the extension only depends on the value of $\varphi$ at points neighboring the stoichiometric surface, over a narrow band of grid points, and it is performed at every Runge-Kutta substage. Therefore, the advection, source, and FHB propagation terms need to be only evaluated at these boundary points, which allows high-order methods to be applied without significantly increasing the computational cost of the method since most points in the domain are skipped.

\section{Tests of the complete algorithm}

The performance of the algorithms was studied using several test cases of increasing degree of complexity. These tests were conducted in addition to several elementary tests that verified that the individual discretization components functioned according to design. For example, advection, extension, compression, etc, were all tested individually. Here, we detail the coupled behavior of the complete method for increasing degree of complexity of $\Sigma_{\mathrm{st}}$.

\subsection{Flat $\Sigma_{\text {st }}$}

The convergence rate of the method is first evaluated using a very smooth initial condition, given by $\varphi=\sin \left(2 \pi x / L_{x}\right)$ (where $L_{x}$ is the length of the domain in the $\mathrm{x}$-direction) advected at $u=1$ over a flat $\Sigma_{\text {st }}$ without compression, see Section 4.1.4. Table 1 shows better than fourth-order convergence rate at lower resolution and convergence to fourth order at the highest resolution in all the norms, as expected for a smooth field. This test verifies the convergence rate of the WENO method.

The second test corresponds to propagation of a circular flame hole in a flat stationary surface. Here, extension is not required because the solution is everywhere parallel to the surface plane (taken as the horizontal plane). The hole is advected at uniform velocity $u=1$ and in consequence the dilatational term of advection is identically zero. This allows us to verify the accuracy of the conservative WENO algorithm when the flame hole formation and self-propagation are also 


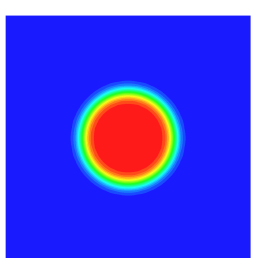

(a) $t=0$

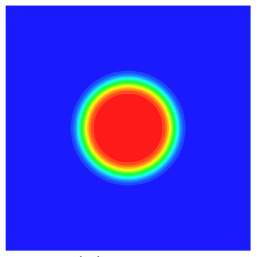

(e) $t=0$

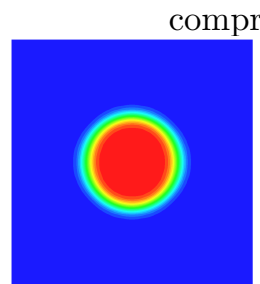

(b) $t=T$

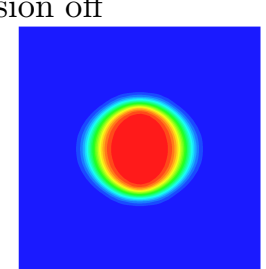

(c) $t=10 T$

compression on

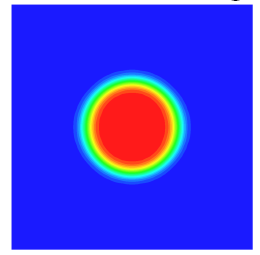

(f) $t=T$

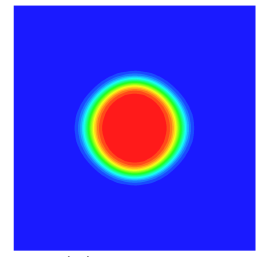

(g) $t=10 T$

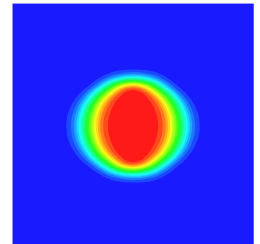

(d) $t=30 T$

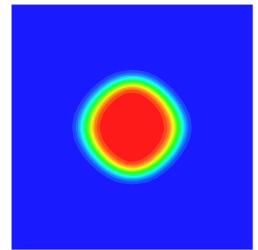

(h) $t=30 T$

Figure 6: Flame state $\varphi$ after one, ten, and thirty flow-through times $T$ for a flat $\Sigma_{\text {st }}$, computed on a $64 \times 48 \times 2$ grid in a domain of size $L_{x}=1.0, L_{y}=0.75, L_{z}=0.03125$. Color scale used is consistent with scale in figure 4 .

disabled. The compression algorithm used $\epsilon_{c}=L_{x} / 64$ and applied only two subiterations with pseudo-time CFL of 0.0025 . Two subiterations of the compression algorithm are quite effective at preventing smearing of $\varphi$ due to numerical dissipation over long time integrations. Figure 6 shows results from this test case, comparing the solution after 1, 10, and 30 flow-through times $T=L_{x} / u$, without and with compression. As can be seen, the compression algorithm limits the effect of diffusion, keeping the interface thin even after a very long time. Since the whole algorithm is a Cartesian-based discretization method, there is some deformation of the shape of the interface over long periods of time which has its roots in the advection algorithm itself, due to grid anisotropy errors, but observe that the thickness of $\varphi$ is unchanged even at late times, as desired.

Table 2 shows the convergence rate of the method. Here, since a very sharp initial condition is used, the WENO method on the coarser meshes exhibits a lower convergence rate of third-order. As the mesh is refined, the method has a higher, third- to fourth-order convergence. As seen in Table 2(b), compression does not significantly affect the convergence when a sufficiently high resolution is used.

\subsection{Sinusoidal $\Sigma_{\mathrm{st}}$}

The third test considers advection of a flame hole in a periodic domain over a $\Sigma_{\text {st }}$ of sinusoidal shape along the direction of a flow with velocity components

$$
\begin{aligned}
& u=\frac{1}{\sqrt{1+\left(a k_{x} \cos \left(k_{x} x\right)\right)^{2}}} \\
& v=0 \\
& w=\frac{-a k_{x} \cos \left(k_{x} x\right)}{\sqrt{1+\left(a k_{x} \cos \left(k_{x} x\right)\right)^{2}}}
\end{aligned}
$$




\begin{tabular}{c|cccc} 
Resolution & $L^{1}$ & $L^{2}$ & $L^{\infty}$ & Order \\
\hline $32 \times 32 \times 2$ & $3.49 \times 10^{-4}$ & $5.27 \times 10^{-3}$ & $1.33 \times 10^{-1}$ & \\
$48 \times 48 \times 2$ & $8.15 \times 10^{-5}$ & $1.93 \times 10^{-3}$ & $7.69 \times 10^{-2}$ & 2.48 \\
$64 \times 64 \times 2$ & $2.82 \times 10^{-5}$ & $7.75 \times 10^{-4}$ & $4.24 \times 10^{-2}$ & 3.15 \\
$96 \times 96 \times 2$ & $5.03 \times 10^{-6}$ & $2.15 \times 10^{-4}$ & $1.75 \times 10^{-2}$ & 3.16 \\
$128 \times 128 \times 2$ & $1.77 \times 10^{-6}$ & $9.25 \times 10^{-5}$ & $9.52 \times 10^{-3}$ & 2.93 \\
$192 \times 192 \times 2$ & $3.22 \times 10^{-7}$ & $2.25 \times 10^{-5}$ & $3.31 \times 10^{-3}$ & 3.49 \\
\multicolumn{5}{|c}{$(\mathrm{a})$} \\
Resolution & $L^{1}$ & $L^{2}$ & $L^{\infty}$ & Order \\
\hline $32 \times 32 \times 2$ & $3.26 \times 10^{-4}$ & $4.98 \times 10^{-3}$ & $1.25 \times 10^{-1}$ & \\
$48 \times 48 \times 2$ & $7.99 \times 10^{-5}$ & $1.69 \times 10^{-3}$ & $6.79 \times 10^{-2}$ & 2.66 \\
$64 \times 64 \times 2$ & $2.30 \times 10^{-5}$ & $6.23 \times 10^{-4}$ & $3.41 \times 10^{-2}$ & 3.48 \\
$96 \times 96 \times 2$ & $3.55 \times 10^{-6}$ & $1.26 \times 10^{-4}$ & $9.30 \times 10^{-3}$ & 3.93 \\
$128 \times 128 \times 2$ & $1.18 \times 10^{-6}$ & $4.71 \times 10^{-5}$ & $3.80 \times 10^{-3}$ & 3.43 \\
$192 \times 192 \times 2$ & $3.16 \times 10^{-7}$ & $1.47 \times 10^{-5}$ & $1.36 \times 10^{-3}$ & 2.89
\end{tabular}

(b)

Table 2: Advection of a flame disc in the $x$-direction on a flat surface without (a) and with (b) compression. Error comparison is between the initial condition and the solution after one flow through time. Order of accuracy is estimated based on the $L^{2}$-norm.

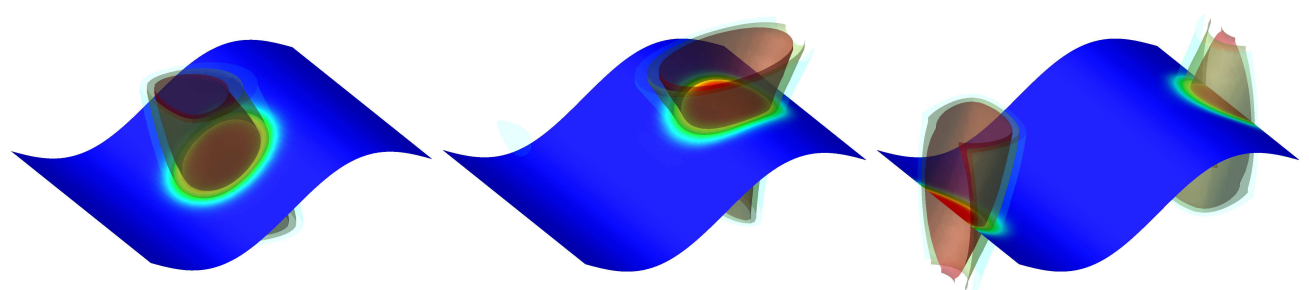

(a) $t=0$

(b) $t=T / 4$

(c) $t=T / 2$

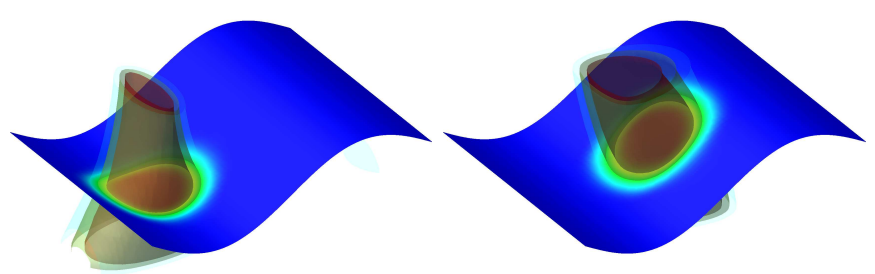

(d) $t=3 / 4 T$

(e) $t=T$

Figure 7: Solution after one flow-through time, $T$, for the advection of a flame disc on a sinusoidal surface with a mesh resolution of $64 \times 48 \times 32$. Color scale used is consistent with scale in figure 4 . 


\begin{tabular}{c|cccc} 
Resolution & $L^{1}$ & $L^{2}$ & $L^{\infty}$ & Order \\
\hline $32 \times 24 \times 16$ & $9.35 .38 \times 10^{-4}$ & $9.92 \times 10^{-3}$ & $2.47 \times 10^{-1}$ & \\
$48 \times 36 \times 24$ & $3.48 \times 10^{-4}$ & $5.17 \times 10^{-3}$ & $1.62 \times 10^{-1}$ & 1.61 \\
$64 \times 48 \times 32$ & $1.56 \times 10^{-4}$ & $2.87 \times 10^{-3}$ & $1.09 \times 10^{-1}$ & 2.05 \\
$96 \times 72 \times 48$ & $4.42 \times 10^{-5}$ & $1.03 \times 10^{-3}$ & $5.17 \times 10^{-2}$ & 2.53 \\
$128 \times 96 \times 64$ & $1.84 \times 10^{-5}$ & $4.78 \times 10^{-4}$ & $2.98 \times 10^{-2}$ & 2.66 \\
$192 \times 144 \times 96$ & $4.03 \times 10^{-6}$ & $1.26 \times 10^{-4}$ & $1.08 \times 10^{-2}$ & 3.29
\end{tabular}

Table 3: Advection of a flame disc in the $x$-direction on a sinusoidal surface. Error comparison is between the initial condition and the solution after one flow through time. Order of accuracy is estimated based on the $L^{2}$-norm.

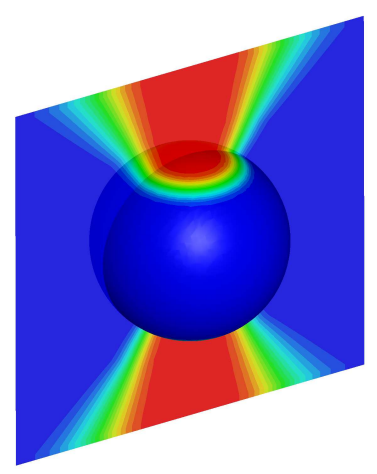

(a) Initial condition

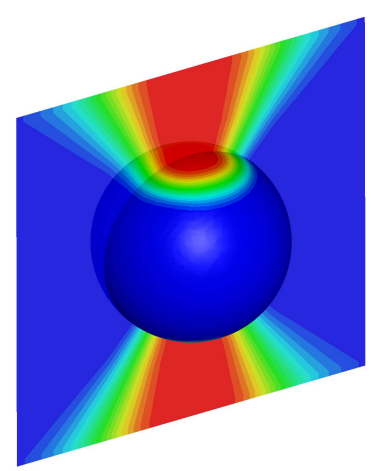

(b) After one rotation

Figure 8: Solution after one flow-through time of a flame disc rotated on a moving sphere by an irrotational vortex with a mesh resolution of $64 \times 64 \times 64$. Color scale used is consistent with scale in figure 4 . 


\begin{tabular}{c|cccc} 
Resolution & $L^{1}$ & $L^{2}$ & $L^{\infty}$ & Order \\
\hline $32 \times 32 \times 32$ & $1.40 \times 10^{-3}$ & $1.28 \times 10^{-2}$ & $2.39 \times 10^{-1}$ & \\
$48 \times 48 \times 48$ & $5.42 \times 10^{-4}$ & $6.43 \times 10^{-3}$ & $1.44 \times 10^{-1}$ & 1.69 \\
$64 \times 64 \times 64$ & $2.33 \times 10^{-4}$ & $3.19 \times 10^{-3}$ & $9.11 \times 10^{-2}$ & 2.44 \\
$96 \times 96 \times 96$ & $7.04 \times 10^{-5}$ & $1.19 \times 10^{-3}$ & $4.52 \times 10^{-2}$ & 2.43 \\
$128 \times 128 \times 128$ & $2.63 \times 10^{-5}$ & $5.10 \times 10^{-4}$ & $2.08 \times 10^{-2}$ & 2.95 \\
$192 \times 192 \times 192$ & $6.15 \times 10^{-6}$ & $1.36 \times 10^{-4}$ & $6.60 \times 10^{-3}$ & 3.26
\end{tabular}

Table 4: Advection of a flame disc by an irrotational vortex on a spherical surface. Error comparison is between the initial condition and the solution after one rotation. Order of accuracy is estimated based on the $L^{2}$-norm.

\begin{tabular}{c|cccc} 
Resolution & $L^{1}$ & $L^{2}$ & $L^{\infty}$ & Order \\
\hline $32 \times 32 \times 32$ & $1.80 \times 10^{-3}$ & $1.53 \times 10^{-2}$ & $2.49 \times 10^{-1}$ & - \\
$48 \times 48 \times 48$ & $6.39 \times 10^{-4}$ & $7.47 \times 10^{-3}$ & $1.65 \times 10^{-1}$ & 1.77 \\
$64 \times 64 \times 64$ & $2.81 \times 10^{-4}$ & $3.83 \times 10^{-3}$ & $1.08 \times 10^{-1}$ & 2.32 \\
$96 \times 96 \times 96$ & $9.11 \times 10^{-5}$ & $1.52 \times 10^{-3}$ & $5.64 \times 10^{-2}$ & 2.27 \\
$128 \times 128 \times 128$ & $3.77 \times 10^{-5}$ & $7.49 \times 10^{-3}$ & $3.52 \times 10^{-2}$ & 2.47 \\
$192 \times 192 \times 192$ & $8.99 \times 10^{-6}$ & $2.14 \times 10^{-4}$ & $1.40 \times 10^{-2}$ & 3.09
\end{tabular}

Table 5: Advection of a flame disc by an irrotational vortex on a moving spherical surface. Error comparison is between the initial condition and the solution after one rotation. Order of accuracy is estimated based on the $L^{2}$-norm.

with $a=0.1$ and $k_{x}=2 \pi / L_{x}$ referring to the amplitude and wavenumber of the sinusoidalwave surface. The flow-through time is calculated as the arc-length along the sine wave and is $T=1.09 L_{x} / u_{0}, u_{0}=1$, for the chosen parameters. The initial value of $\varphi$ is given by the intersection of a cylinder of radius $0.1875 L_{x}$ with $\Sigma_{\text {st }}$, creating a "flame disc" on the surface. Advection, compression and extension subalgorithms are enabled in this calculation and the dilatational-like term discretization is also active since the velocity field is not divergence free, making this is a more realistic test. After one $T$, the shape of the hole should return unmodified to the initial condition because self-propagation of the FHB and hole formation are disabled. Figure 7 shows the evolution of the extended $\varphi$ field at several instants in $0 \leq t \leq T$. The initial condition and the solution after one flow-through time are compared quantitatively in terms of the one-, two-, and infinity norms of the difference between the initial condition and the solution after one flow-through time around $\Sigma_{\text {st }}$. These error norms are tabulated in Table 3.

\subsection{Spherical $\Sigma_{\text {st }}$}

The rotation of flame discs on a spherical $\Sigma_{\mathrm{st}}$ is tested to ensure the accuracy on a closed surface with variable flow velocity. Circular flame disc regions $(\varphi=1)$ are initialized on $\Sigma_{\text {st }}$ and are then extended to create the initial condition for this test case. $\varphi$ is advected by an irrotational vortex with velocity given by $\mathbf{u}^{*}=(u, v, w)=(0,-z, y)$, which rotates the flame disc on the surface. The complete algorithm is used to evolve $\varphi$ but, as before, without self-propagation of FHB or flame hole formation. After one rotation, the solution is compared with the initial condition, which should be ideally identical, as shown in Figure 8. The accuracy results are given in Table 4.

To test the algorithm on a moving $\Sigma_{\mathrm{st}}$, the previous test is modified such that the sphere moves laterally in a sinusoidal motion in time. The center of the sphere, initially at the origin of the domain, 


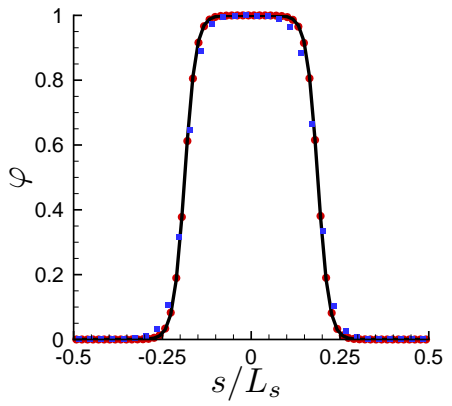

(a) Flat

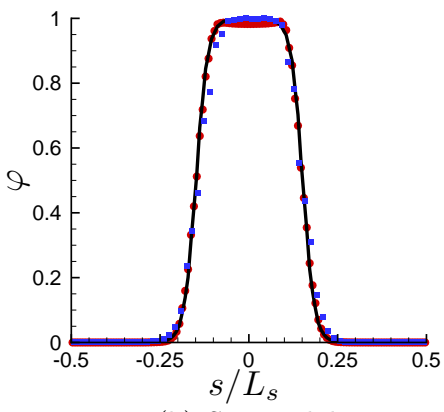

(b) Sinusoidal

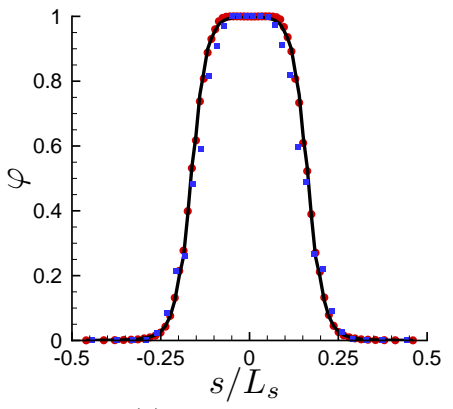

(c) Moving sphere

Figure 9: One-dimensional cuts for the flat, sinusoidal, and spherical test cases. $s(x)=x$ for the flat case, $s=$ $\int_{0}^{x} \sqrt{1+\left(k_{x} a\right)^{2} \cos ^{2}\left(k_{x} x^{\prime}\right)} d x^{\prime}$ for the sinusoidal surface, and $s=\arcsin (y / R)$ for the spherical surface. $L_{s}$ is the total length of the surface in the $x$-direction for each case. Circles denote a test case with twice the resolution of the squares. The exact result is represented by a black curve.

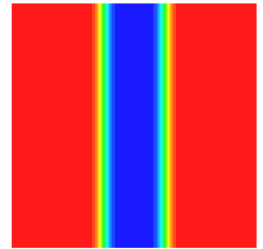

(a) Initial condition

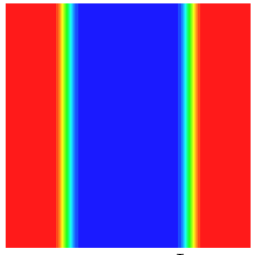

(b) $t=\frac{1}{8} \frac{L_{x}}{V_{e, \max }}$

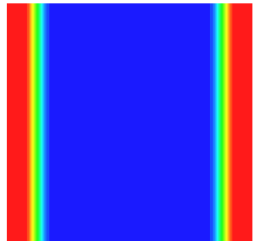

(c) $t=\frac{1}{4} \frac{L_{x}}{V_{e, \max }}$

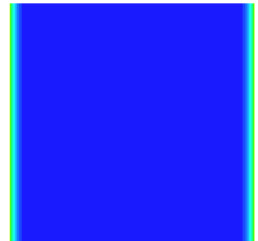

(d) $t=\frac{3}{8} \frac{L_{x}}{V_{e, \max }}$

Figure 10: Evolution of a flame hole strip expanding at maximum negative edge speed, $-V_{e, \max }$, at different instants of time. Color scale used is consistent with scale in figure 4.

moves in the $x$-direction as $x_{c}(t)=a \sin (2 t)$, making the surface velocity $\mathbf{V}_{\text {st }}=(2 a \cos (2 t), 0,0)$. The velocity of the flow is then determined according to $\mathbf{u}=\mathbf{u}^{*}+\mathbf{V}_{\mathrm{st}}$, where $\mathbf{u}^{*}$ is the velocity in the flow in the stationary sphere case used above. The amplitude of the displacement used in the test is $a=0.125 L_{x}$. The movement of $\Sigma_{\text {st }}$ makes it such that the closest point transform and extension matrix must be recreated at every subiteration of the Runge-Kutta, including the determination of the boundary points of the surface. The accuracy results are given in Table 5 .

Figure 9 shows one-dimensional cuts of the solution for the flat, sinusoidal and spherical surface test cases at two different resolutions. For the sinusoidal and spherical surface test cases, resolutions of $N_{x}=64$ and $N_{x}=128$, where $N_{x}$ denotes the number of grid points in the $x$-direction, are chosen. As the method is significantly more accurate for the flat surface case, a reduced resolution of $N_{x}=32$ and $N_{x}=64$ is chosen to visually show the distinction between the theoretical and numerical results. The values are linearly interpolated to the surface along the $z$-direction in each case. 


\begin{tabular}{c|cccc} 
Resolution & $L^{1}$ & $L^{2}$ & $L^{\infty}$ & Order \\
\hline $32 \times 32 \times 2$ & $8.22 \times 10^{-4}$ & $8.87 \times 10^{-3}$ & $1.66 \times 10^{-1}$ & \\
$48 \times 48 \times 2$ & $3.16 \times 10^{-4}$ & $4.33 \times 10^{-3}$ & $1.10 \times 10^{-1}$ & 1.77 \\
$64 \times 64 \times 2$ & $1.38 \times 10^{-4}$ & $2.38 \times 10^{-3}$ & $7.85 \times 10^{-2}$ & 2.08 \\
$96 \times 96 \times 2$ & $2.98 \times 10^{-5}$ & $7.72 \times 10^{-4}$ & $4.18 \times 10^{-2}$ & 2.78 \\
$128 \times 128 \times 2$ & $1.06 \times 10^{-5}$ & $3.64 \times 10^{-4}$ & $2.32 \times 10^{-2}$ & 2.61 \\
$192 \times 192 \times 2$ & $2.40 \times 10^{-6}$ & $1.10 \times 10^{-4}$ & $1.06 \times 10^{-2}$ & 2.95
\end{tabular}

Table 6: Test of self-advection for the closing flame hole test case, compared with the exact solution at $t=0.125 \frac{L_{x}}{S_{L}}$. Order of accuracy is estimated based on the $L^{2}$-norm.

\subsection{Flame hole propagation and creation tests}

To test the FHB propagation properties, a flame hole strip was initialized in the domain and allowed to move without a background flow velocity. As described in Appendix A.1, the scalar dissipation was set to $\chi=2 \chi_{\mathrm{q}}$, such that the maximum negative edge flame speed, $-V_{e, \max }$, is reached. The strip is initialized to have a half-width of $\frac{1}{8} L_{x}$ on a $128 \times 128 \times 2$ domain. Under these circumstances, we expect the $\varphi=0.5$-level to reach the edge of the computational domain at $\frac{3}{8} \frac{L_{x}}{V_{e, \max }}$, which corresponds to the observed behavior in figure 10. The opposite case, where the flame hole heals under a low scalar dissipation, was also tested. A flame hole is initialized in a domain of zero scalar dissipation with zero flow velocity and allowed to heal. Now, according to the FHB propagation model Eq. (A.1), the edge flame travels at a speed of $S_{L}$. The hole is initialized to be $0.25 L_{x}$ in radius and at a constant speed is expected to heal over a time $0.25 L_{x} / S_{L}$ on a $128 \times 128 \times 2$ domain. The flame is seen to be closing at the appropriate time, with the $\varphi=0.5$ level reaching the center of the domain at the expected time, the hole closing and the shape being nearly circular throughout the evolution, see figure 11 . The solution is compared with the exact solution at $t=0.125 L_{x} / S_{L}$, where the hole has reduced to a radius of $0.125 L_{x}$. Accuracy results are tabulated in Table 6 .

Finally, a test of flame hole formation was considered where a cylindrical region of high scalar dissipation, greater than $\chi_{\mathrm{q}}$, was set in a circular region of $0.25 L_{x}$ radius at the center of a $64 \times$ $64 \times 2$ domain with $\varphi$ set initially to one. Figure 12 shows the formation of a cylindrical hole in approximately $\tau_{\mathrm{q}}$ corresponding to the high scalar dissipation region and the permanence of this hole in time.

As the complexity of the algorithm increases, the order of accuracy decreases from $5^{\text {th }}$-order for the advection of a smooth initial condition on a flat surface at a constant speed to $3^{\text {rd }}$-order for the complete algorithm. The accuracy of the complete algorithm is limited by the order of accuracy of the treatment of the nonlinear FHB propagation term and the closest point extension, both of which use third-order accurate discretizations (since the extension uses quadratic interpolation). A simpler closest point extension, using linear interpolation, would limit the accuracy of the method further.

\section{Applications to realistic turbulent flow}

In the first part, we will apply the flame hole dynamics algorithm to show the evolution of the flame state field in a complicated stoichiometric surface extracted from an existing DNS. Here, 


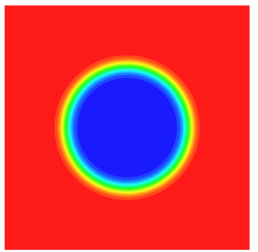

(a) Initial condition

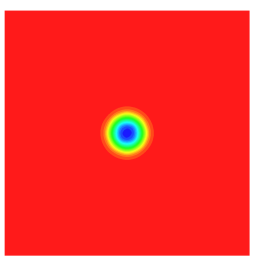

(d) $t=\frac{3}{16} \frac{L_{x}}{S_{L}}$

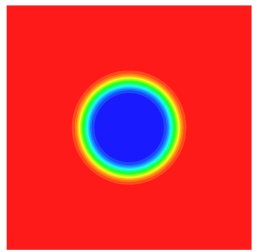

(b) $t=\frac{1}{16} \frac{L_{x}}{S_{L}}$

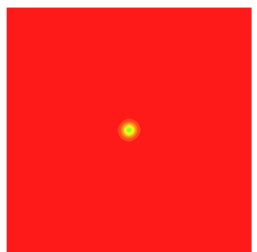

(e) $t=\frac{1}{4} \frac{L_{x}}{S_{L}}$

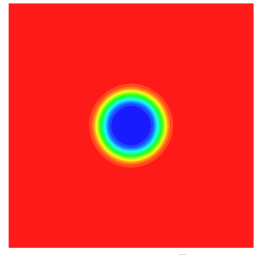

(c) $t=\frac{1}{8} \frac{L_{x}}{S_{L}}$

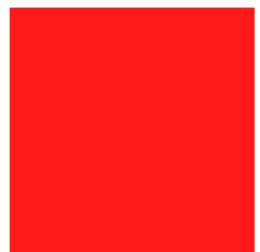

(f) $t=\frac{5}{16} \frac{L_{x}}{S_{L}}$

Figure 11: A flame hole closing in a domain with zero scalar dissipation. The hole is expected to close at $0.25 L_{x} / S_{L}$. Color scale used is consistent with scale in figure 4.

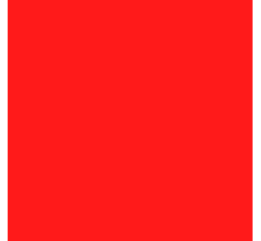

(a) Initial condition

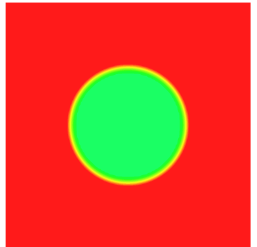

(b) $t=\frac{1}{3} \tau_{\mathrm{q}}$

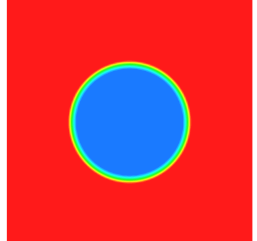

(c) $t=\frac{2}{3} \tau_{\mathrm{q}}$

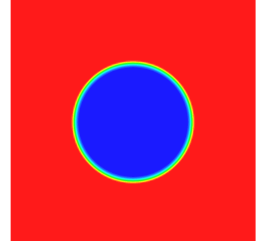

(d) $t=\tau_{\mathrm{q}}$

Figure 12: A hole forming in the presence of a cylindrical region of high scalar dissipation beyond the quenching limit, $\chi_{\mathrm{q}}$. Color scale used is consistent with scale in figure 4 . 


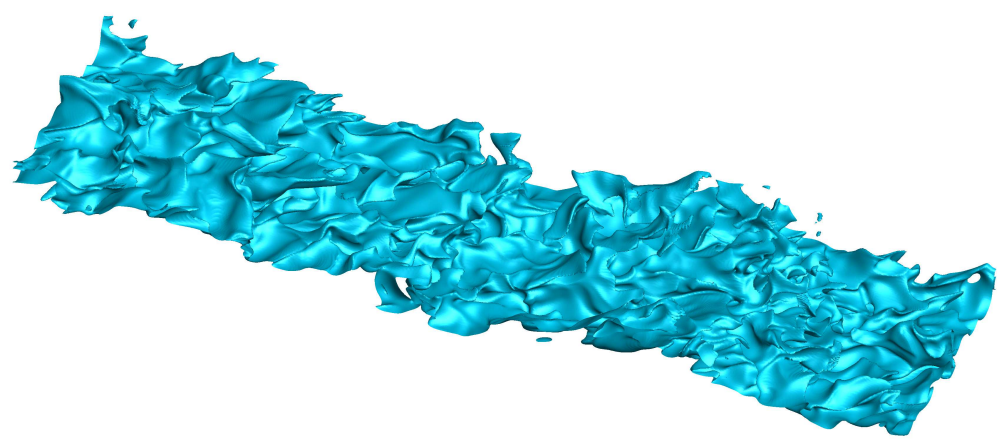

Figure 13: View of stoichiometric surface in a turbulent reacting shear layer obtained from DNS.

there is no coupling with the flow. This tests showcase the ability of the algorithm to function in a really complicated surface. In the second part, we showcase a fully coupled simulation where flame hole dynamics and flow interact consistently.

\subsection{Simulation using a given mixture fraction field}

In more realistic applications of flame hole dynamics, the stoichiometric surface is highly distorted by the stirring and mixing effects of the flow. To assess the applicability of the flame hole dynamics algorithms to more realistic conditions, we tested the performance of the method on the stoichiometric surface obtained from a DNS of a reacting turbulent shear layer at a fixed instant of time, see [67]. The simulation used a Burke-Schumann approximation for the chemistry, evaluating the density (which varies in space) through the ideal gas law for the mixture and it does not include extinction or flame holes.

The flame state is initialized to $\varphi=1$ in the $Z_{-}<Z<Z_{+}$band and flame holes are allowed to develop on the extracted stoichiometric surface, according to the velocity and scalar dissipation fields extracted from the DNS at the fixed instant in time. Figure 13 shows a three-dimensional rendering of $Z=Z_{\text {st }}$ from the DNS. This simulation is described in detail in the reference, but its key properties are: turbulence Reynolds number of $R e_{\lambda, p e a k}=82$ at the time we applied flame hole dynamics, grid size of $768 \times 258 \times 192$, model combustion of methane with air at $Z_{\text {st }}=0.2$.

Figure 14 shows the probability-density-function (PDF) of velocity and scalar dissipation at $\Sigma_{\text {st }}$, highlighting the broad variation of different fields around the flame surface. Note that the PDF of scalar dissipation, in particular, is shown in logarithmic coordinates due to the large variation of extreme high (and low) values of $\chi$ on the stoichiometric surface (since it is a highly intermittent quantity). The flame hole dynamics algorithm was used on this case with the fixed flow velocity and mixture fraction provided by the DNS. The particular values of the crossover, $\chi_{0}$ and quenching, $\chi_{\mathrm{q}}$, scalar dissipation used in the edge flame velocity model, Eq. (A.1), were chosen to trigger partial extinction of the flame thoughout the domain. These are indicated in figure 14(b), where $\chi_{0} /\langle\chi\rangle=1$, and $\chi_{\mathrm{q}} /\langle\chi\rangle=2.86$ (resulting in roughly a third of the stoichiometric surface being below 


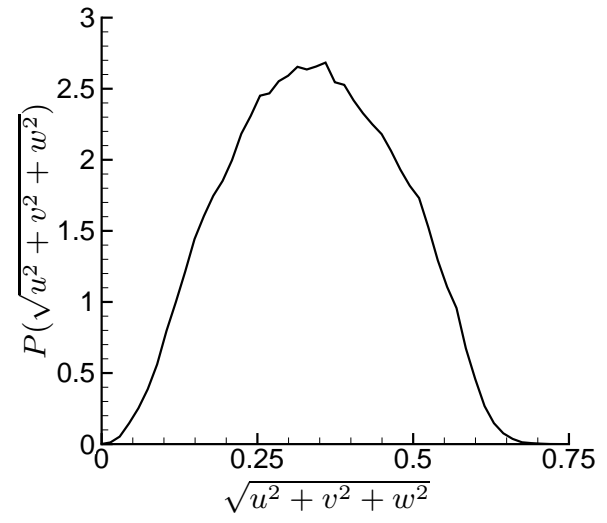

(a) Kinetic energy

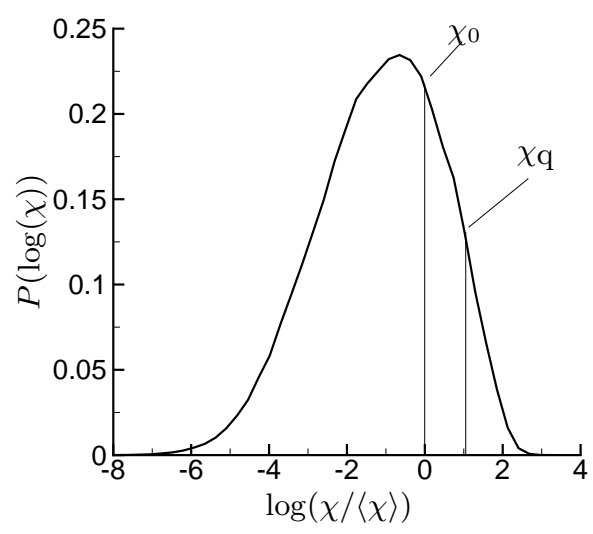

(b) Scalar dissipation

Figure 14: Statistics of the shear layers on the stoichiometric surface.

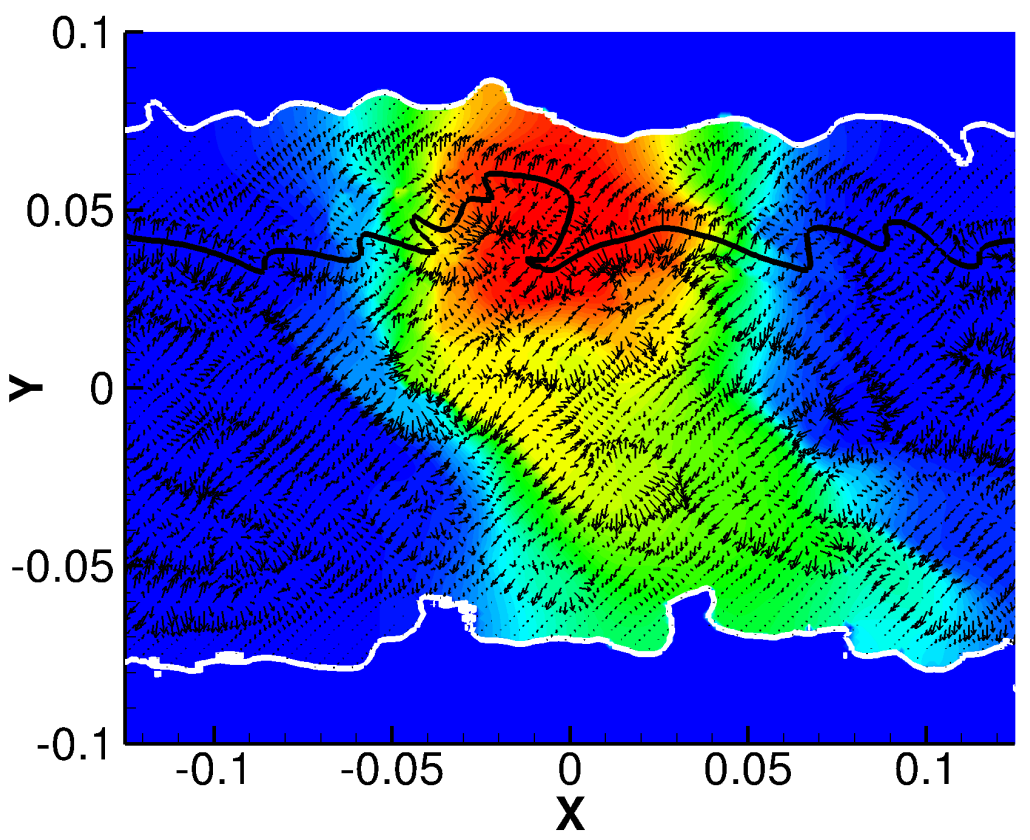

(a) Extended flame disc

Figure 15: A slice of the extended $\varphi$ applied to a turbulent shear layer DNS [68], with the stoichiometric surface shown as a thick continuous black curve and the direction field a throughout the domain. The exterior boundary is shown through a thick, continuous white curve. The vector field represents the advection velocity a of the mixture fraction field. Color scale used is consistent with scale in figure 4. 


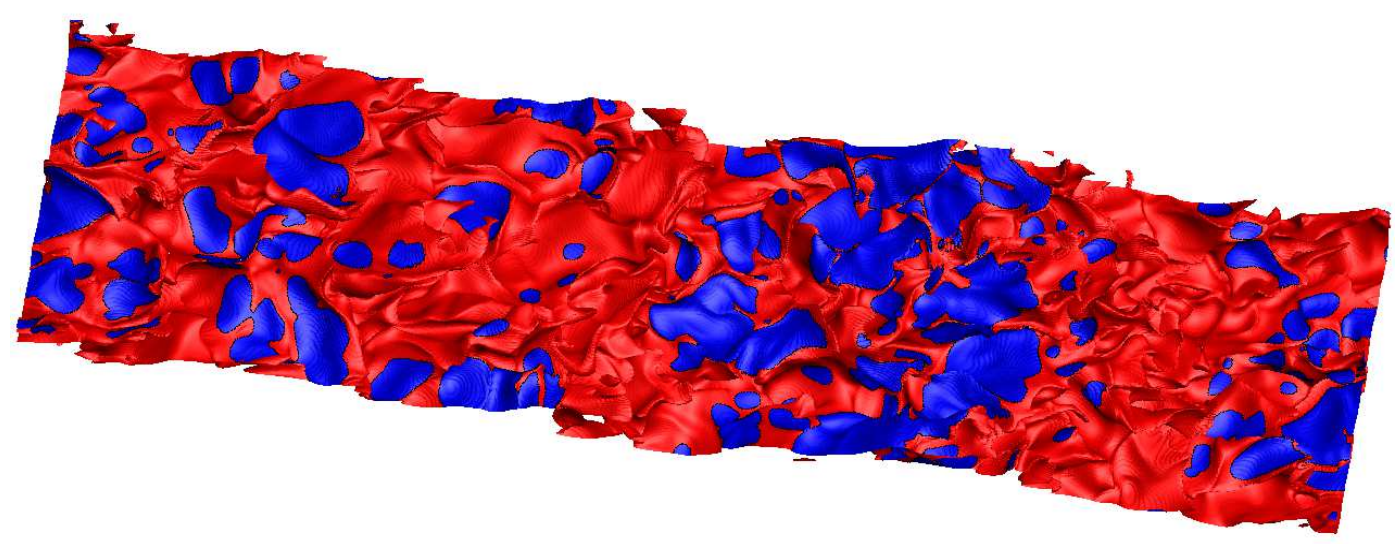

(a) $t \approx 0.01 L_{x} / \Delta u$

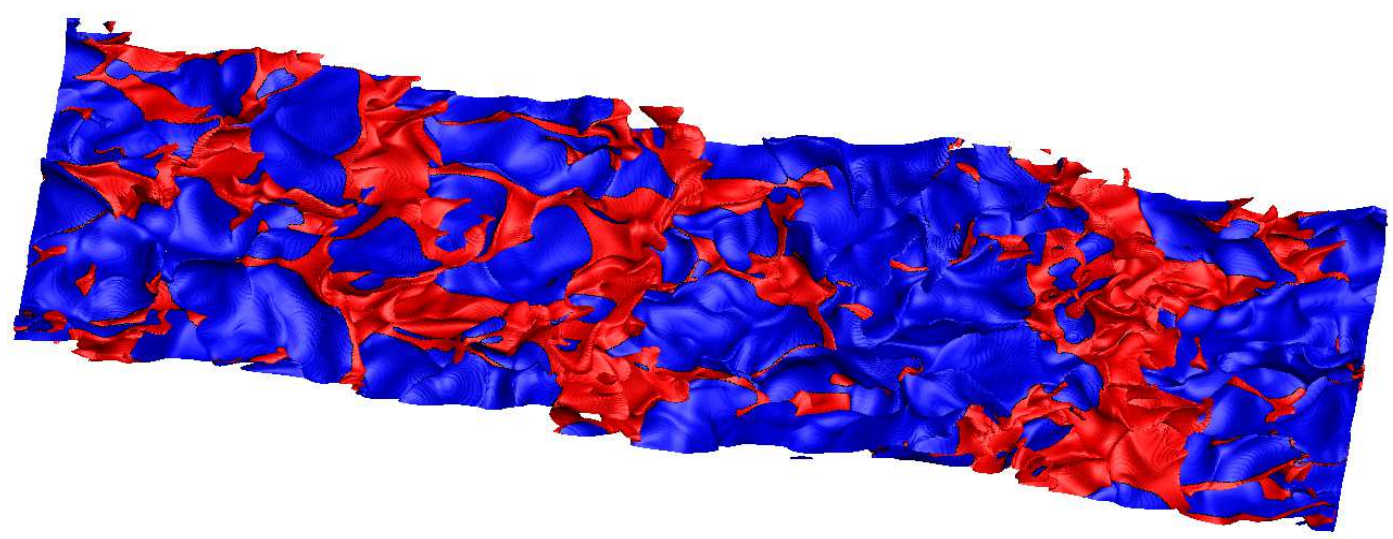

(b) $t \approx 1.0 L_{x} / \Delta u$

Figure 16: Flame hole formation and evolution on $\Sigma_{\text {st }}$ from a shear layer DNS. Blue regions correspond to "flame holes." 
the quenching dissipation, with a ramp parameter of $\Delta_{\chi} /\langle\chi\rangle=0.2$, see Eq. (A.2)), where angle brackets denote the mean value of $\chi$ on $\Sigma_{\text {st }}$. A flame hole quenching time of $\tau_{\mathrm{q}}=4.0 \times 10^{-3} L_{x} / \Delta u$, where $\Delta u$ is the difference between the free stream velocities in the shear layer, is used so that flame holes form quickly relative to the advection and FHB propagation time scales. The FHB propagation uses $V_{e}(0)=0.1 \Delta u \approx 0.3\langle u\rangle$ and a maximum negative flame speed of $V_{e, \max }=-3 V_{e}(0)$ so that the advection and FHB propagation occur at similar time scales. The flame state, $\varphi$, at the stoichiometric surface is propagated into the domain by the extension operation, giving information about the gas composition at any point between the upper and lower exterior boundaries of the shear layer. Through numerical experimentation, a reference value of extension dissipation of $\varepsilon_{0}=$ $1.0 \times 10^{-4}$ with characteristic length scale $\ell=10\left(\delta_{\omega} / \Delta u\right)^{1 / 2}$, defined in Section 4.2 where $\delta_{\omega}$ is the vorticity thickness, see [67], was found to be sufficient for rapid convergence of the multigrid solver of the extension algorithm. Critical points are detected by comparing with a value of $g_{\epsilon}=0.05|\nabla Z|_{\text {avg }}$ (averaged over the domain of extension), with the Robin boundary condition parameterized by $\alpha_{R}=0.25 /\left(\varepsilon_{0}(1+\ell / \Delta)\right)$. The interface is compressed with a subiteration CFL of 0.0025 and $\epsilon_{c}=2.0 \min (\Delta x, \Delta y, \Delta z)$. Figure 15 shows a two-dimensional cut of the extended $\varphi$ for a section of 3D turbulent shear layer containing both critical points and exterior boundaries. The values on $\Sigma_{\text {st }}$ were initialized as a flame disc through the center of the shear layer and they are extended normal to the iso-surfaces of $Z$ by the extension algorithm. Note that the extension algorithm does not always propagate the value of $\varphi$ all the way to the shear layer boundaries because of the three-dimensional nature of $\Sigma_{\text {st }}(\nabla Z$ has a component off the plane shown in figure 15).

Figure 16 shows the solution of the flame hole dynamics model Eq. (33) at two instants in time for the shear layer, with $\varphi$ initialized to be 1 everywhere on $\Sigma_{\text {st }}$. Flame holes form in regions where the scalar dissipation exceeds the quenching dissipation and expand due to FHB propagation and the flow velocity until an equilibrium is reached where the FHB propagation is counter balanced by the flow velocity $\left(\left|d \mathbf{r}_{i} / d t\right|=\mathbf{u} \cdot \mathbf{m}+V_{e}=0\right.$; see Eq. (8)). Flame holes, flame islands, and flame strips all develop and propagate on the complicated and realistic $\Sigma_{\text {st }}$.

\subsection{Fully coupled algorithm in a lifted turbulent jet flame simulation.}

The present method was implemented in "Nalu," an open source fluid dynamics code from Sandia National Laboratories [69]. The code uses a low Mach number formulation based on the asymptotic expansion of Majda and Sethian [70] and a control-volume finite element discretization. Advection terms are discretized using a mixed second-order upwind and fourth-order accurate discretization with a smoothness detection algorithm to blend the stencils accordingly. Diffusion terms use a centered fourth-order accurate discretization in space and time integration uses the implicit first-order Euler scheme. The implicit system of equations is solved using a parallel GMRES solver with an algebraic multigrid preconditioner for the pressure Poisson equation and a GaussSeidel preconditioner for the other equations (e.g. momentum, mixture fraction) [71]. The solver is designed for extreme scale computation, with demonstrated weak scaling up to nine billion elements on 131,000 cores [69].

The computational domain was designed to simulate Sandia's piloted methane-air jet flame series (see [72] for a description of the experiments). In these flames, the main jet, which has a diameter of $d=0.72 \mathrm{~cm}$, is composed of methane diluted with air in 1:3 proportion by volume, resulting in $Z_{\mathrm{st}}=0.3557$. The coflow and jet enter the burner at ambient temperature while the pilot (made of burned $\mathrm{C}_{2} \mathrm{H}_{2}$ ) has a temperature of $1880 \mathrm{~K}$. The computational domain is conical with a length of $100 d$, a minimum radius of $10 d$ and a maximum radius of $40 d$. The pipe inlet sits $15 d$ below the reference plane $y=0$. In the present demonstration simulation, the bulk jet velocity 

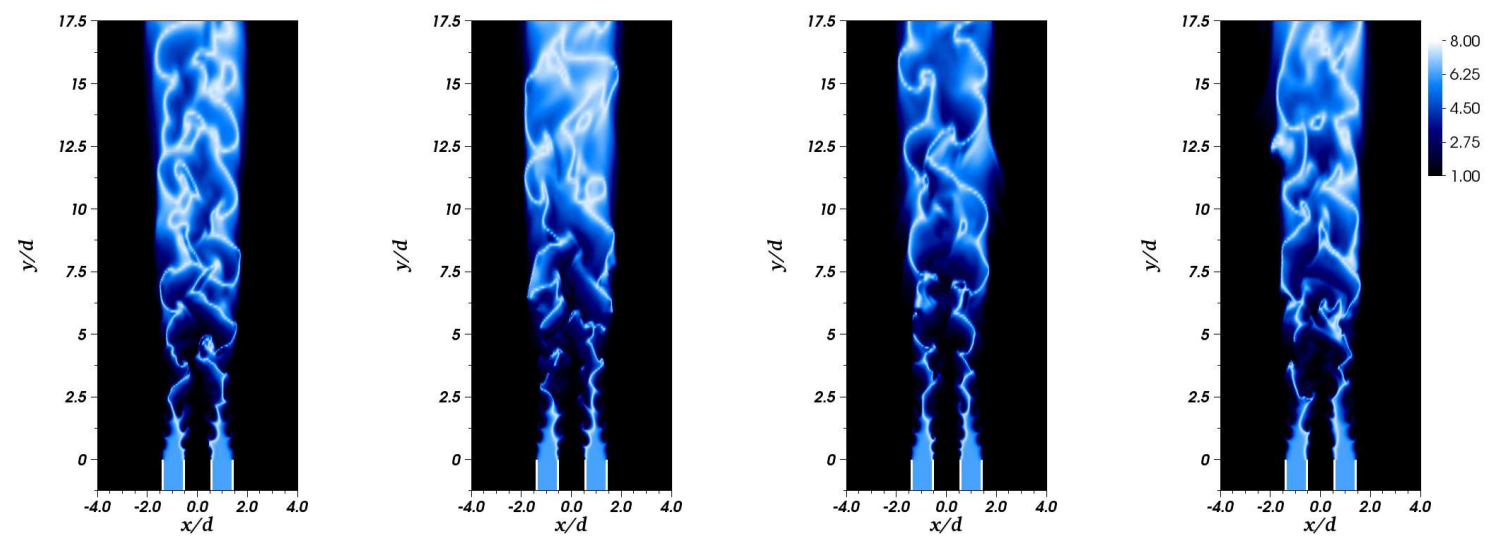

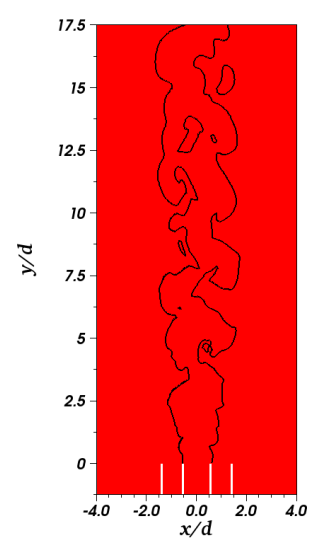

(e) $t=0$

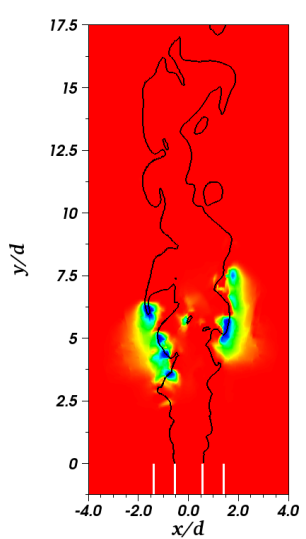

(f) $t \approx 5 d / U_{\text {jet }}$

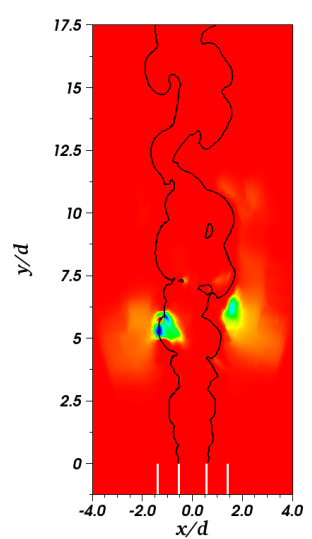

(g) $t \approx 15 d / U_{\text {jet }}$

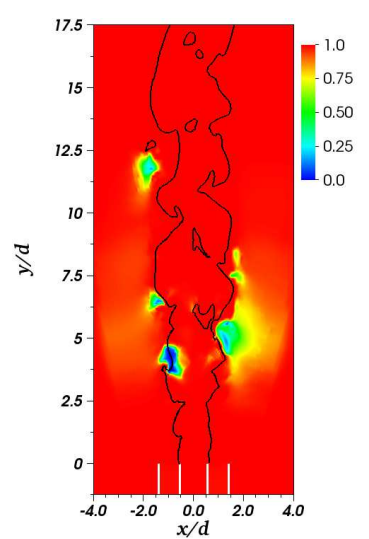

(h) $t \approx 25 d / U_{\text {jet }}$

Figure 17: Cross section of temperature normalized by the free-stream temperature of $T_{o}=298 \mathrm{~K}$ in a turbulent reacting jet simulation using flame hole dynamics to approximate extinction at several instants (top panel); adiabatic flame temperature is $7.78 T_{o}$. Cross section of flame state field at the corresponding times (bottom panel); black curve denotes the stoichiometric surface and blue regions denote extinction.

is $U_{\text {jet }}=20 \mathrm{~m} \mathrm{~s}^{-1}$ while the pilot velocity is $U_{\text {pilot }}=4.5 \mathrm{~m} \mathrm{~s}^{-1}$. The corresponding jet Reynolds number based on the bulk velocity is approximately 9,000 . The simulation used to demonstrate the flame hole dynamics has approximately 6 million cells and a mesh spacing of $d / \Delta x \approx 80$ in the core region of the flow. The turbulence in this test simulation is not expected to be fully resolved (which does not affect the flame hole dynamics algorithm itself). This test shows the applicability of the method with a complicated, time-evolving, stoichiometric surface with coupling between the flame state and the flow (full variable density).

The chemistry in the flame has been simplified to a Burke-Schumann model with two mixture fractions, $Z$ and $\hat{Z}$, describing the main jet and the pilot [73], respectively. The flame state is coupled to the thermochemical state of the gas through a two-state rule between a non-reacting mixing solution $\left\{Y_{i}^{\mathrm{m}}(Z, \hat{Z}), T^{\mathrm{m}}(Z, \hat{Z})\right\}$ (linear in $Z$ ) and the flame solution $\left\{Y_{i}^{\mathrm{f}}(Z, \hat{Z}), T^{\mathrm{f}}(Z, \hat{Z})\right\}$, where the subscript $i$ denotes the chemical species index and $T$ denotes temperature, giving the 
overall mixture composition and temperature from

$$
Y_{i}=(1-\varphi) Y_{i}^{\mathrm{m}}(Z, \hat{Z})+\varphi Y_{i}^{\mathrm{f}}(Z, \hat{Z}), \quad T=(1-\varphi) T^{\mathrm{m}}(Z, \hat{Z})+\varphi T^{\mathrm{f}}(Z, \hat{Z}) .
$$

The density is then obtained from the ideal gas equation of state

$$
\rho=\frac{p_{0}}{R^{0} T(Z, \hat{Z}, \varphi)} \sum \frac{Y_{i}(Z, \hat{Z}, \varphi)}{W_{i}},
$$

where $R^{0}$ denotes the ideal gas constant, $p_{0}$ the atmospheric pressure, and $W_{i}$ the molecular weight of the species. A more complete description in terms of flamelets would make $Y_{i}^{\mathrm{f}}$ and $T^{\mathrm{f}}$ also a function of $\chi$ in Eq. (84), but it does not alter the flow-coupling formulation. An entrainment condition based on Batchelor's jet solution is applied at the cylindrical boundary of the domain with the entrainment given by [74]. Azimuthal disturbances of $20 \%$ of the peak jet velocity are added at the inlet of the pipe in order to attain a turbulent jet flow at its outlet into the main domain, $y>0$.

The edge flame speed was also modeled with the simple law given by Eq. (A.1) using approximate parameters appropriate for this chemistry. A value of $\chi_{q}=1000 \mathrm{~s}^{-1}$ was chosen as the quenching value, which is a rounded value of the real quenching dissipation for this mixture [37], and the crossover scalar dissipation was chosen to be $\chi_{0}=2 / 3 \chi_{q}$. The peak edge flame speed was set to $V_{e}(0)=2.0 \mathrm{~m} \mathrm{~s}^{-1}$ (similar to the value reported in Phillips [75]) and the quenching time was set to be $200 \mu \mathrm{s}$. The anti-dissipative correction parameters were set to $\epsilon_{c}=0.05 \mathrm{~mm}$ (of the order of the cell size in the main pipe of the jet), $\mathcal{U}_{0}=20 \mathrm{~m} \mathrm{~s}^{-1}$ (equal to the jet bulk velocity), and the subiteration CFL was set to 0.00001 .

Figure 17 shows results of the coupled simulation where we observe two-dimensional cuts of $T$ and $\varphi$ through the center of the three-dimensional domain at several times. The flame is initialized to be burning everywhere, although the strain in some regions near the pipe ending is too large to sustain the flame. Therefore, flame holes appear on the stoichiometric surface in regions of large scalar dissipation, above the quenching value, expand in regions where the scalar dissipation is greater than the crossover dissipation, are advected downstream, and heal in regions of low scalar dissipation (dynamically).

Figure 18 shows a three-dimensional rendering of the instantaneous stoichiometric surface colored by the scalar dissipation and flame state at two instants during the simulation. In these figures the flow goes from left to right (diagonally). Regions of high scalar dissipation (above $\chi_{q}$ ) are distributed throughout the near-nozzle region in a chaotic manner. It can be seen that extinction takes place once the scalar dissipation becomes large enough but the flame holes (after being created) have their own dynamics and may remain open even after the scalar dissipation decreases in magnitude. They are advected downstream and eventually heal (over regions of much lower scalar dissipation). This dynamic character of the quenched regions is a key signature of flame holes which cannot be linked instantaneously with the local values of $\chi$. A phenomenology that flame hole dynamics tries to describe.

Although comparing the FHD algorithm with a simulation without modeling extinction/reignition is not physically consistent (since they are not describing the same physics), we have obtained some estimates from these simulations. A companion simulation without flame hole modeling, which is nearly optimally implemented in Nalu, was observed to be about twice as fast as the FHD fully coupled algorithm. This suggest that the present algorithm is quite competitive, although we did not devote any special effort to ensure that the implementation of FHD in Nalu is as efficient as the corresponding algorithms without flame holes. 


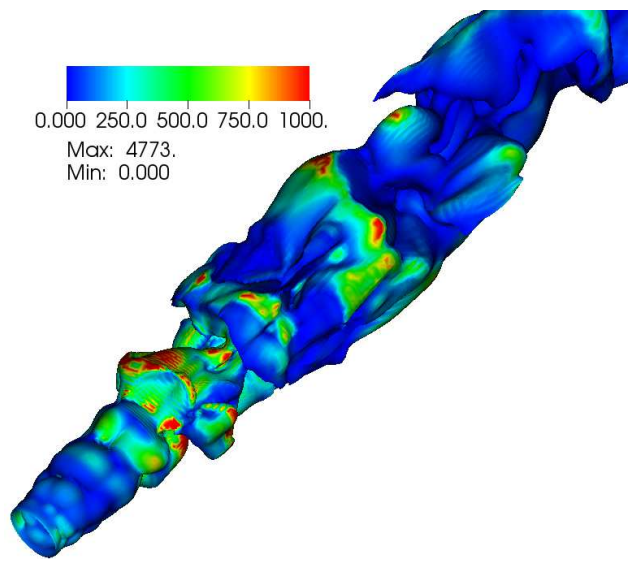

(a) $\chi$ at $t \approx 5 d / U_{\text {jet }}$

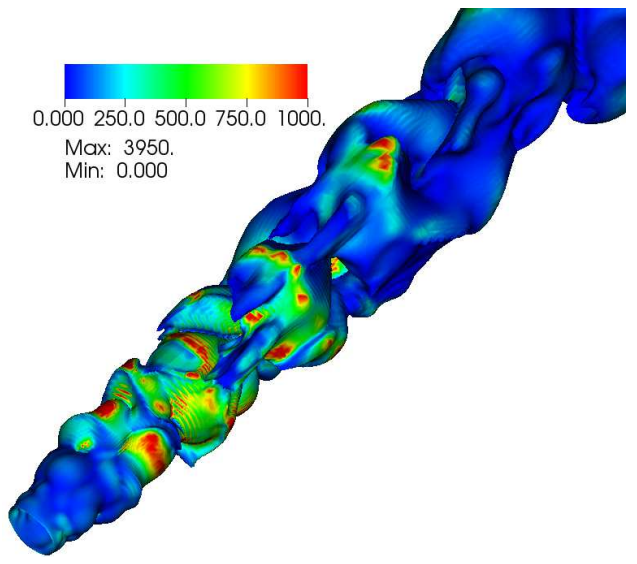

(c) $\chi$ at $t \approx 25 d / U_{\text {jet }}$

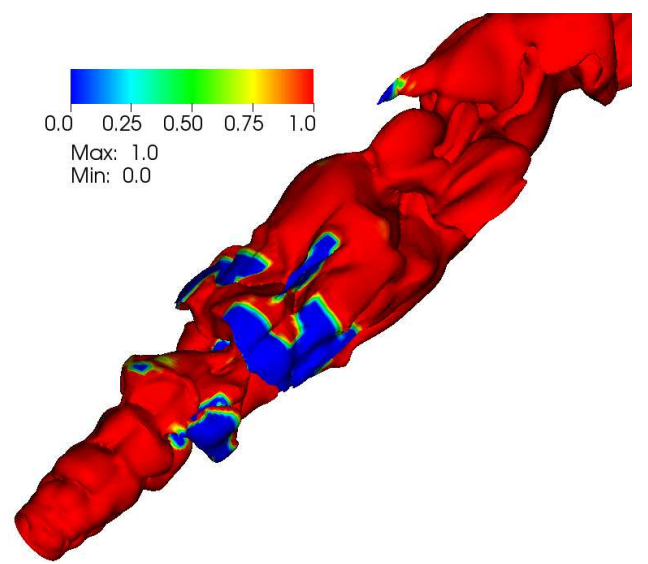

(b) $\varphi$ at $t \approx 5 d / U_{\text {jet }}$

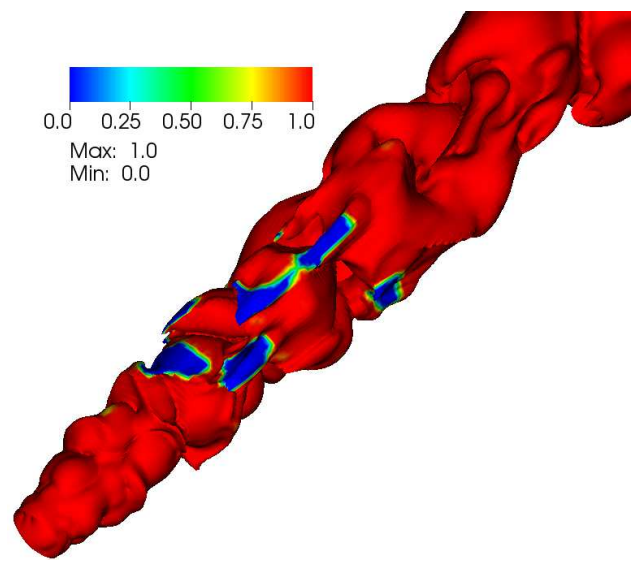

(d) $\varphi$ at $t \approx 25 d / U_{\text {jet }}$

Figure 18: Scalar dissipation (left) and flame state (right) on the stoichiometric surface at times of $t \approx 5 d / U_{\text {jet }}$ and $25 d / U_{\text {jet }}$. The color range for $\chi$ has been capped at $\chi_{q}$ for clarity. 


\section{Conclusion}

The paper describes the development of a new numerical approach for the equations of diffusion flame hole dynamics. The main challenge is the need to solve an evolution equation, of the flame state field, defined on a moving complex surface. Here, a Cartesian embedding technique is preferred because it renders the algorithm very efficient when the underlying grid is structured. Because the flame state field does not represent a material property, it is a dynamical description of the flame burning conditions, the advection part of the governing equation is not in conservation form. This necessitates a specialized treatment where advection is split into divergence- and dilatational-like terms. The former is discretized using an existing high-order finite-volume WENO method while the latter uses a new high-order Gaussian quadrature method developed specifically for this application, which is made consistent with the WENO method at the level of the reconstruction of the field. The choice of high-order method is dictated by the need to minimize anisotropic errors tied to the logically Cartesian mesh, since flame hole dynamics is a highly geometrical process. Another key component of the algorithm is an extension procedure whereby the flame state field that is nominally defined around the stoichiometric surface is extended to cover the whole three-dimensional domain. This allows the determination of the gas mixture composition anywhere in space. The extension algorithm is formulated by determining the stationary solution of a propagation equation that is solved using a multigrid technique. Furthermore, we demonstrate the combined integration of the different mechanisms present in flame hole dynamics (advection, flame boundary propagation, and flame quenching) and their successful numerical coupling. The method is shown to be accurate on several test geometries and was employed to solve for the evolution of flame holes on a realistic and complex turbulent stoichiometric surface taken from a DNS of a shear layer with a mesh involving millions of grid points. A demonstration of a full turbulent lifted jet diffusion flame simulation shows applicability to real laboratory flames.

\section{Acknowledgements}

This work was supported in part by the National Science Foundation under grant CBET-1236164 and by the Department of Energy, National Nuclear Security Administration, under Award Number(s) DE-NA0002374.

\section{Appendix A. Constitutive models}

\section{Appendix A.1. Edge flame velocity model}

The edge flame velocity depends primarily on the rate of strain of the flow (here parameterized by the mixture fraction rate of dissipation) and the cold-to-hot mixture density ratio. It is customary to normalize the edge flame velocity by its limiting value at $\chi=0, V_{e}(0)$, which is proportional to the laminar premixed speed of a mixture at the stoichiometric conditions for the mixture just ahead of the edge flame [24] times an enhancement factor due to thermal expansion [32]. Owing to this dependence on strain rate, the main diffusion flame can undergo reignition by the healing of the flame hole boundary through a positive edge-flame velocity (and correspondingly a low scalar dissipation) or further extinction by growth of the flame hole, when the edge-flame velocity is negative due to large local values of scalar dissipation. It is also possible, but not considered here, for a quenched zone to become reactive due to the folding of burning regions near non-burning regions of the stoichiometric surface $[12,30]$. 
The edge flame velocity is provided through a model as suggested in Pantano and Pullin [28]. To date, there are limited theoretical studies providing precise calculations of the edge flame velocity as a function of the relevant local flow conditions and mixture conditions for real chemistry and realistic levels of heat release, (most studies concentrate on the thermo-diffusive limit where density is constant [76]). This is a consequence of the complicated and difficult-to-solve nature of the twodimensional fully coupled reactive Navier-Stokes boundary value problem. Despite these limitations, and only for the purpose of a formulation of a flame hole dynamics framework, one can approximate the edge flame velocity, according to

$$
\frac{V_{e}}{V_{e}(0)}=1-\frac{1+\frac{1}{\frac{x}{x q}-1}}{1+\frac{1}{\frac{x}{x q}-1}}
$$

where $\chi_{0}$ is the value of the scalar dissipation for which the edge speed is zero.

\section{Appendix A.2. Flame extinction model}

Computationally, it is advantageous to model the extinction process by assuming it takes place over a finite amount of time, $\tau_{\mathrm{q}}$, as explained in Hewson [37]. If we impose that complete extinction is reached at $t=\tau_{\mathrm{q}}$ (starting from $t=0$ ) following the time-dependent law, $\varphi(t)=\left(1-t / \tau_{\mathrm{q}}\right)^{2}$, then one can determine the form of the sink, $\dot{Q}(\varphi, \chi)$, to be

$$
\dot{Q}(\varphi, \chi)=R\left(\chi-\chi_{\mathrm{q}}\right) \frac{2 \sqrt{\varphi}}{\tau_{\mathrm{q}}}
$$

where $R\left(\chi-\chi_{\mathrm{q}}\right)$ is a ramp function,

$$
R\left(\chi-\chi_{\mathrm{q}}\right)= \begin{cases}0 & \chi<\chi_{\mathrm{q}}, \\ \frac{\chi-\chi_{\mathrm{q}}}{\Delta_{\chi}} & \chi_{\mathrm{q}} \leq \chi<\chi_{\mathrm{q}}+\Delta_{\chi} \\ 1 & \chi \geq \chi_{\mathrm{q}}+\Delta_{\chi}\end{cases}
$$

and $\Delta_{\chi}$ is a parameter set to be small relative to $\chi_{\mathrm{q}}$. This model can be enhanced, and made more accurate, by replacing $R\left(\chi-\chi_{\mathrm{q}}\right)$ with the scalar dissipation impulse model of Hewson [37].

\section{Appendix B. Discretization details}

Appendix B.1. MUSTA

The multistage upwind method (called MUSTA, for "multi stage") is an iterative approximate Riemann solver used in the conservative part of the finite-volume technique. It solves the Riemann problem between cell interfaces on a local, coarse space-time mesh $(d-\tau)$ using the "First-ORder CEntered (FORCE) flux". The FORCE flux is an average of the Lax-Friedrichs flux,

$$
F_{1 / 2}^{L F}\left(\varphi^{L}, \varphi^{R}\right)=\frac{1}{2}\left[F\left(\varphi^{L}\right)+F\left(\varphi^{R}\right)\right]-\frac{1}{2} \frac{\Delta d}{\Delta \tau}\left[\varphi^{R}-\varphi^{L}\right],
$$

and the two-stage Lax-Wendroff flux,

$$
F_{1 / 2}^{L W}\left(\varphi^{L}, \varphi^{R}\right)=\varphi_{1 / 2}=\frac{1}{2}\left[\varphi^{L}+\varphi^{R}\right]-\frac{1}{2} \frac{\Delta \tau}{\Delta d}\left[F\left(\varphi^{L}\right)-F\left(\varphi^{R}\right)\right]
$$


The method allows for the use of a local Courant number, based on the local speed in the direction normal to the face. The local timestep is

$$
\Delta \tau=C F L \frac{\Delta d}{S}
$$

where $S$ is the local wavespeed decided in our case by the velocity $u$ as $\left|\left(\max \left(u^{L}, u^{R}\right)\right)\right|$. Transmissive boundary conditions are used in the local mesh. For instance, with two cells, the transmissive boundary is

and the intercell flux is

$$
F_{-1 / 2}=\varphi^{L} \quad F_{3 / 2}=\varphi^{R}
$$

$$
F_{1 / 2}=\frac{1}{2} F_{1 / 2}^{L F}\left(\varphi^{L}, \varphi^{R}, \Delta \tau, \Delta d\right)+\frac{1}{2} F_{1 / 2}^{L W}\left(\varphi^{L}, \varphi^{R}, \Delta \tau, \Delta d\right) .
$$

As reported by Titarev and Toro [52], two cells produce accuracy similar to the HLL (Harten, Lax, van Leer) flux in the case of the Euler equations. Greater detail into this method is provided in a series of papers $[54,55,56]$.

\section{Appendix B.2. Weights and abscissae of the modified Gaussian quadrature}

The nonconservative weights and abscissae were calculated for a two-point $(m=2)$ and threepoint $(m=3)$ quadrature of $\varphi$ (three and four-point quadrature of the velocity, correspondingly). For $m=2$, the weights are

$$
W=\left(\begin{array}{ccc}
-\frac{1}{2}-\frac{1}{\sqrt{3}} & \frac{2}{\sqrt{3}} & \frac{1}{2}-\frac{1}{\sqrt{3}} \\
-\frac{1}{2}+\frac{1}{\sqrt{3}} & -\frac{2}{\sqrt{3}} & \frac{1}{2}+\frac{1}{\sqrt{3}}
\end{array}\right)
$$

and the abscissae are $x_{\alpha}=\{-1 / \sqrt{3}, 1 / \sqrt{3}\}$ for the scalar and $x_{b}=\{-1,0,1\}$ for the velocity. For $m=3$,

$$
\begin{array}{ll}
W_{11}=-\frac{5(1849+\sqrt{43(69437-1520 \sqrt{15})})}{14706}, & W_{12}=\frac{5(1849+\sqrt{43(69437+1520 \sqrt{15})})}{14706} \\
W_{13}=\frac{5(-1849+\sqrt{43(69437-1520 \sqrt{15})})}{14706}, & W_{14}=-\frac{5(-1849+\sqrt{43(69437+1520 \sqrt{15}})}{14706} \\
W_{21}=-\frac{4(67 \sqrt{215}-1247)}{7353}, & W_{22}=-\frac{4(1247+67 \sqrt{215})}{7353} \\
W_{23}=\frac{4(1247+67 \sqrt{215})}{7353} & W_{24}=\frac{4(67 \sqrt{215}-1247)}{7353} \\
W_{31}=\frac{5(-1849+\sqrt{43(69437+1520 \sqrt{15})})}{14706} & W_{32}=-\frac{5(-1849+\sqrt{43(69437-1520 \sqrt{15}})}{14706} \\
W_{33}=-\frac{5(-1849+\sqrt{43(69437-1520 \sqrt{15})})}{14706} & W_{34}=\frac{5(1849+\sqrt{43(69437-1520 \sqrt{15})})}{14706}
\end{array}
$$

with abscissae

$$
\begin{aligned}
& x_{\alpha}=\left\{-\sqrt{\frac{3}{5}}, 0, \sqrt{\frac{3}{5}}\right\} \text { and } \\
& x_{\beta}=\left\{-\frac{1}{20}(5+\sqrt{215}),-\frac{1}{20}(-5+\sqrt{215}), \frac{1}{20}(-5+\sqrt{215}), \frac{1}{20}(5+\sqrt{215})\right\} .
\end{aligned}
$$




\section{Appendix B.3. WENO interpolation and reconstruction}

A combined WENO interpolation and reconstruction is used to determine the flux across each face of the conservative term of the governing equation [77]. The interpolation and reconstruction steps are dimensionally-split, using a one-dimensional interpolation in each cardinal direction to evaluate the variable of interest at the specified Gaussian quadrature collocation point. The conservative method requires the evaluation of the scalar field, $\varphi$, and the velocity, $\mathbf{u}$, at Gauss points located on the faces of the finite-volume cell. The nonconservative method requires the evaluation of $\mathbf{u}$ and $\varphi$ at separate points prescribed by a modified Gaussian quadrature discussed in Section 4.1. The scalar field is volume-averaged as part of the finite-volume procedure and requires WENO reconstruction whereas the velocity field is assumed to be given as pointwise values from some exterior flow solver and requires WENO interpolation.

A $5^{\text {th }}$-order WENO interpolation is developed by defining an interpolating polynomial of degree 4 through a centered stencil of five points, $S=\left\{u_{i-2}, \ldots, u_{i+2}\right\}$. The stencil is broken into three substencils: $S_{-1}=\left\{u_{i-2}, u_{i-1}, u_{i}\right\}, S_{0}=\left\{u_{i-1}, u_{i}, u_{i+1}\right\}$, and $S_{+1}=\left\{u_{i}, u_{i+1}, u_{i+2}\right\}$. Interpolating polynomials are constructed for each of these stencils. Weights are chosen such that the value of subpolynomials matches the wide polynomial. These linear weights are then modified such that a greater importance is given on subpolynomials with lower total variation, providing robustness for problems containing sharp gradients. The subpolynomials are

$$
\begin{aligned}
p_{-1} & =u_{i-2}\left[\frac{1}{2} \xi(1+\xi)\right]-u_{i-1}[\xi(2+\xi)]+u_{i}\left[\frac{1}{2}(1+\xi)(2+\xi)\right] \\
p_{0} & =u_{i-1}\left[\frac{1}{2} \xi(-1+\xi)\right]+u_{i}\left[1-\xi^{2}\right]+u_{i+1}\left[\frac{1}{2}(1+\xi)(2+\xi)\right] \\
p_{+1} & =u_{i}\left[\frac{1}{2}(-2+\xi)(-1+\xi)\right]-u_{i+1}[\xi(-2+\xi)]+u_{i+2}\left[\frac{1}{2} \xi(-1+\xi)\right],
\end{aligned}
$$

and the wide stencil is

$$
\begin{aligned}
p_{\text {wide }}= & u_{i-2}\left[\frac{1}{24}(\xi+1) \xi(\xi-1)(\xi-2)\right]-u_{i-1}\left[\frac{1}{6}(\xi+2) \xi(\xi-1)(\xi-2)\right] \\
& +u_{i}\left[\frac{1}{4}(\xi+2)(\xi+1)(\xi-1)(\xi-2)\right] \\
& -u_{i+1}\left[\frac{1}{6}(\xi+2)(\xi+1) \xi(\xi-2)\right]+u_{i+2}\left[\frac{1}{24}(\xi+2)(\xi+1)(\xi-1)\right] .
\end{aligned}
$$

The linear weights are chosen such that, at the desired point $\xi$, the sum of subpolynomials matches the wide polynomial:

$$
p_{\text {wide }}(\xi)=\sum_{j=-1}^{1} \gamma_{j} p_{j}(\xi) .
$$

Solving the resulting linear system, the linear weights, $\gamma$, are defined at the location $\xi$ :

$$
\begin{aligned}
\gamma_{-1} & =\frac{1}{12}(\xi-2)(\xi-1), \\
\gamma_{0} & =-\frac{1}{6}(\xi-2)(\xi+2), \quad \text { and } \\
\gamma_{+1} & =\frac{1}{12}(\xi+1)(\xi+2) .
\end{aligned}
$$


Nonlinear weights are then constructed with the intent to approximate the values of the linear weights in regions of the domain that are smooth, achieving maximal $5^{\text {th }}$-order of accuracy in the interpolation, while increasing dissipation in regions where the field contains sharp gradients and, correspondingly, the decreasing the order of accuracy of the method in such regions to at worst $2^{\text {nd }}$. The nonlinear weights are developed based on the spatial variation of points in the stencil,

$$
\beta_{l}=\sum_{m=1}^{r-1} \Delta \xi^{2 m-1} \int_{\xi_{i-1 / 2}}^{\xi_{i+1 / 2}}\left(\frac{d^{m}}{d x^{m}} p_{l}(\xi)\right)^{2} d \xi .
$$

and so the nonlinear weight $\omega_{k}$ corresponding to the $k$ subpolynomial is

$$
\alpha_{k}=\frac{\gamma_{j}}{\left(\epsilon_{\mathrm{WENO}}+\beta_{j}\right)^{2}}, \quad \text { and } \quad \omega_{k}=\frac{\alpha_{k}}{\sum_{j=-1}^{1} \alpha_{j}} .
$$

The WENO reconstruction follows a very similar derivation to the WENO interpolation. With WENO reconstruction, it is assumed that the variable is known only on a cell-averaged basis, like what is given by solving for the variable through a finite-volume discretization. The WENO interpolation procedure is undertaken with a surrogate primitive variable,

$$
U\left(x_{i+1 / 2}\right)=\int_{x_{\min }}^{x_{i+1 / 2}} u(\xi) d \xi=\sum_{l=0}^{i} \Delta x \bar{u}_{l} .
$$

Here, $x_{\min }$ is a fixed number chosen to correspond with the left-most boundary of the domain. As we know the pointwise values of $U$, we can perform the WENO interpolation, with the value of $u$ obtained through the relationship $u(x)=U^{\prime}(x)$. The subpolynomials are then derived for the 5th-order WENO at a point $x=\xi$,

$$
\begin{aligned}
p_{-1} & =u_{i-2}\left[\frac{1}{24}(-1+12 \xi(1+\xi))\right]+u_{i-1}\left[\frac{1}{12}-\xi(2+\xi)\right]+u_{i}\left[\frac{1}{24}(23+12 \xi(3+\xi))\right] \\
p_{0} & =u_{i-1}\left[\frac{1}{24}(-1+12 \xi(-1+\xi))\right]+u_{i}\left[\frac{13}{12}-\xi^{2}\right]+u_{i+1}\left[\frac{1}{24}(-1+12 \xi(1+\xi))\right] \\
p_{+1} & =u_{i}\left[\frac{1}{24}(23+12 \xi(-3+\xi))\right]+u_{i+1}\left[\frac{1}{12}-\xi(-2+\xi)\right]+u_{i+2}\left[\frac{1}{24}(-1+12 \xi(-1+\xi))\right]
\end{aligned}
$$

The wide polynomial, in the general case of $\xi$, is defined similarly,

$$
\begin{aligned}
p_{\text {wide }}= & u_{i-2}\left[\frac{1}{1920}(9+40(-1+\xi) \xi(-5+2(-1+\xi) \xi))\right] \\
+ & u_{i-1}\left[\frac{1}{480}(-29+20 \xi(-17+2 \xi(9-2(-1+\xi) \xi)))\right] \\
& +u_{i}\left[\frac{1067}{960}-\frac{11}{8} \xi^{2}+\frac{1}{4} \xi^{4}\right] \\
& +u_{i+1}\left[\frac{1}{480}(-29+20 \xi(17-2 \xi(-9+2(1+\xi) \xi)))\right] \\
& +u_{i+2}\left[\frac{1}{1920}(9+40(1+\xi) \xi(-5+2(1+\xi) \xi))\right] .
\end{aligned}
$$


The wide polynomial is constructed in the same manner as the subpolynomials, for a degree 4 polynomial interpolant, yielding the same $5^{\text {th }}$-order accuracy as the interpolation procedure. The linear weights are

$$
\begin{aligned}
\gamma_{-1} & =\frac{9+200 \xi-120 \xi^{2}-160 \xi^{3}+80 \xi^{4}}{80(-1+12 \xi(1+\xi))} \\
\gamma_{0} & =\frac{49-4548 \xi^{2}+5360 \xi^{4}-960 \xi^{6}}{40-6720 \xi^{2}+5760 \xi^{4}} \\
\gamma_{+1} & =\frac{9-200 \xi-120 \xi^{2}+160 \xi^{3}+80 \xi^{4}}{80(-1+12 \xi(-1+\xi))}
\end{aligned}
$$

To reconstruct the derivative, we use the relationship that $u^{\prime}(x)=U^{\prime \prime}(x)$ to construct the subpolynomials,

$$
\begin{aligned}
p_{-1} & =u_{i-2}\left[\frac{1}{2}+\xi\right]+u_{i-1}[-2(1+\xi)]+u_{i}\left[\frac{3}{2}+\xi\right] \\
p_{0} & =u_{i-1}\left[-\frac{1}{2}+\xi\right]+u_{i}[-2 \xi]+u_{i+1}\left[\frac{1}{2}+\xi\right] \\
p_{+1} & =u_{i}\left[-\frac{3}{2}+\xi\right]+u_{i+1}[2(1-\xi)]+u_{i+2}\left[-\frac{1}{2}+\xi\right] .
\end{aligned}
$$

The wide polynomial, in the general case of $\xi$, is defined similarly,

$$
\begin{aligned}
p_{\text {wide }}= & u_{i-2}\left[\frac{1}{48}(1-2 \xi)(5+4 \xi(1-\xi)]\right. \\
+ & u_{i-1}\left[\frac{1}{24}(-17+4 \xi(9+(3-4 \xi) \xi))\right] \\
& +u_{i}\left[-\frac{11}{4}+\xi^{3}\right] \\
+ & u_{i+1}\left[\frac{1}{24}(17-4 \xi(-9+(3+4 \xi) \xi))\right] \\
+ & u_{i+2}\left[\frac{1}{48}(1+2 \xi)(-5+4 \xi(1+\xi))\right] .
\end{aligned}
$$

The wide polynomial is constructed in the same manner as the subpolynomials, for what is now a degree 3 polynomial interpolant, yielding a reduced $4^{\text {th }}$-order accuracy. The linear weights for this reconstruction are

$$
\begin{aligned}
\gamma_{-1} & =\frac{5-6 \xi-12 \xi^{2}+8 \xi^{3}}{24(1+2 \xi)} \\
\gamma_{0} & =\frac{7-48 \xi^{2}+16 \xi^{4}}{12\left(1-4 \xi^{2}\right)} \\
\gamma_{+1} & =\frac{5+6 \xi-12 \xi^{2}-8 \xi^{3}}{24(1-2 \xi)} .
\end{aligned}
$$

The nonlinear weights require the smoothness indicators of the first-order subpolynomials. The 
smoothness indicators are

$$
\begin{aligned}
\beta_{-1} & =\left(u_{i-2}-2 u_{i-1}+u_{i}\right)^{2} \\
\beta_{0} & =\left(u_{i-1}-2 u_{i}+u_{i+1}\right)^{2} \\
\beta_{+1} & =\left(u_{i}-2 u_{i+1}+u_{i+2}\right)^{2} .
\end{aligned}
$$

In this reconstruction, the possibility exists that some of the linear weights are negative. The presence of the negative linear weights is a source of instability [78]. These cases require special treatment. The reconstruction in the event of negative weights is split,

$$
\tilde{\gamma}_{i}^{+}=\frac{1}{2}\left(\gamma_{i}+\theta\left|\gamma_{i}\right|\right), \quad \tilde{\gamma}_{i}^{-}=\tilde{\gamma}_{i}^{+}-\gamma_{i}
$$

with $i$ ranging between -1 and 1 . The value of $\theta$ is taken as $\theta=3$ in accordance to [78]. These values are scaled,

$$
\sigma^{ \pm}=\sum_{j=-1}^{1} \tilde{\gamma}_{j}^{ \pm}, \quad \gamma_{i}^{ \pm}=\tilde{\gamma}_{i}^{ \pm} / \sigma^{ \pm} .
$$

The wide polynomial is then

$$
p_{\text {wide }}(x)=\sigma^{+} p^{+}-\sigma^{-} p^{-},
$$

with the split polynomials

$$
p_{\text {wide }}^{ \pm}(x)=\sum_{j=-1}^{1} \gamma_{j}^{ \pm} p_{j}(x) .
$$

The split wide polynomials, $p_{\text {wide }}^{ \pm}(x)$, can then be used to define linear weights individually, yielding positive linear weights for both of the two split polynomials. 
[1] A. Ratz, A. Voigt, PDE's on surfaces - a diffuse interface approach, Commun. Math. Sci. 4 (2006) 575-590.

[2] M. Bertalmı, L. Cheng, S. Osher, G. Sapiro, Variational problems and partial differential equations on implicit surfaces, Journal of Computational Physics 174 (2001) 759-780.

[3] J. Greer, An improvement of a recent Eulerian method for solving PDEs on general geometries, J. Sci. Comp. 29 (2006) 321-352.

[4] D. Adalsteinsson, J. Sethian, Transport and diffusion of material quantities on propagating interfaces via level set methods, Journal of Computational Physics 185 (2003) 271-288.

[5] J. Xu, H. Zhao, An Eulerian formulation for solving partial differential equations along a moving interface, Journal of Scientific Computing 19 (2003) 573-594.

[6] S. Ruuth, B. Merriman, A simple embedding method for solving partial differential equations on surfaces, J. Comput. Phys. 227 (2008) 1943-1961.

[7] C. Macdonald, S. Ruuth, Level set equations on surfaces via the closest point method, Journal of Scientific Computing 35 (2008) 219-240.

[8] C. Macdonald, J. Brandman, S. J. Ruuth, Solving eigenvalue problems on curved surfaces using the closest point method, Journal of Computational Physics 230 (2011) 7944-7956.

[9] C. Piret, The orthogonal gradients method: A radial basis functions method for solving partial differential equations on arbitrary surfaces, Journal of Computational Physics 231 (2012) 46624675 .

[10] S. Leung, J. Lowengrub, H. Zhao, A grid based particle method for solving partial differential equations on evolving surfaces and modeling high order geometrical motion, Journal of Computational Physics 230 (2011) 2540-2561.

[11] A. Liñán, The asymptotic structure of counterflow diffusion flames for large activation energies, Acta Astronautica 1 (1974) 1007-1039.

[12] J. Hewson, A. Kerstein, Local extinction and reignition in nonpremixed turbulent $\mathrm{CO} / \mathrm{H}_{2} / \mathrm{N}_{2}$ jet flames, Combustion science and technology 174 (2002) 35-66.

[13] V. S. Santoro, A. Liñán, A. Gomez, Propagation of edge flames in counterflow mixing layers: experiments and theory, Proceedings of the Combustion Institute 28 (2000) 2039-2046.

[14] G. Amantini, J. H. Frank, A. Gomez, Experiments on standing and traveling edge flames around flame holes, Proceedings of the Combustion Institute 30 (2005) 313-321.

[15] L. Vervisch, T. Poinsot, Direct numerical simulation of non-premixed turbulent flames, Annual review of fluid mechanics 30 (1998) 655-691.

[16] F. Williams, S. Murthy, Turbulent mixing in nonreactive and reactive flows, Complex Chemical Reaction Systems," SNB Murphy, Ed.(Plenum, New York, 1975) (1975) 189.

[17] N. Peters, Laminar diffusion flamelet models in non-premixed turbulent combustion, Progress in Energy and Combustion Science 10 (1984) 319-339. 
[18] N. Peters, Laminar flamelet concepts in turbulent combustion, Proc. Combust. Inst. 21 (1986) $1231-1250$.

[19] S. Cheatham, M. Matalon, A general asymptotic theory of diffusion flames with application to cellular instability, J. Fluid Mech. 414 (2000) 105-144.

[20] M. Cha, P. D. Ronney, Propagation rates of nonpremixed edge flames, Combustion and Flame 146 (2006) $312-328$.

[21] C. Pantano, Direct simulation of non-premixed flame extinction in a methane-air jet with reduced chemistry, Journal of Fluid Mechanics 514 (2004) 231-270.

[22] L. Wang, E. R. Hawkes, J. H. Chen, Flame edge statistics in turbulent combustion, Proceedings of the Combustion Institute 33 (2011) 1439-1446.

[23] V. Favier, L. Vervisch, Edge flames and partially premixed combustion in diffusion flame quenching, Combustion and flame 125 (2001) 788-803.

[24] J. Buckmaster, Edge-flames, Progress in Energy and Combustion Science 28 (2002) 435-475.

[25] M. Matalon, Flame dynamics, Proceedings of the Combustion Institute 32 (2009) 57-82.

[26] J. W. Dold, L. J. Hartley, D. Green, Dynamics of laminar triple-flamelet structures in nonpremixed turbulent combustion, Dynamical Issues in Combustion Theory, The IMA Volumes in Mathematics and its Applications (1991).

[27] J. W. Dold, L. J. Hartley, D. Green, Dynamics of laminar triple-flamelet structures in nonpremixed turbulent combustion, Dynamics of hetergeneous combustion and reacting systems 152 (1993) 70-86.

[28] C. Pantano, D. I. Pullin, A statistical description of turbulent diffusion flame holes, Combustion and Flame 137 (2004) $295-305$.

[29] J. Kim, S. H. Chung, K. Y. Ahn, J. S. Kim, Simulation of a diffusion flame in turbulent mixing layer by the flame hole dynamics model with level-set method, Combustion Theory and Modelling 10 (2006) 219-240.

[30] K.-P. Liao, C. Pantano, A numerical study of reignition induced by a diffusion flame, Combustion Theory and Modelling 13 (2009) 1025-1052.

[31] F. Williams, Combustion theory, Addison-Wesley Pub., Reading, MA, 1985.

[32] G. Ruetsch, L. Vervisch, A. Liñán, Effects of heat release on triple flames, Physics of Fluids 7 (1995) 1447-1454.

[33] J. Daou, A. Linan, The role of unequal diffusivities in ignition and extinction fronts in strained mixing layers, Combustion Theory and Modelling 2 (1998) 449-477.

[34] V. Kurdyumov, M. Matalon, Effect of thermal expansion on the stabilization of an edge-flame in a mixing-layer model, 2008. Submitted for publication. 
[35] K. Liao, M. Matalon, C. Pantano, A flow pattern that sustains an edge flame in a straining mixing layer with finite thermal expansion, Proceedings of the Combustion Institute in press. (2014).

[36] B. Cuenot, F. Egolfopoulos, T. Poinsot, An unsteady laminar flamelet model for non-premixed combustion, Combustion Theory and Modelling 4 (2000) 77-97.

[37] J. C. Hewson, An extinction criterion for nonpremixed flames subject to brief periods of high dissipation rates, Combustion and Flame (2013).

[38] A. Dziubek, Equations for two-phase flows: a primer, Meccanica (2011) 1-18.

[39] T. März, C. Macdonald, Calculus on surfaces with general closest point functions, arXiv preprint arXiv:1202.3001 (2012).

[40] L. Simon, Lectures on geometric measure theory, 1984.

[41] M. P. Do Carmo, Differential geometry of curves and surfaces, volume 2, Prentice-Hall Englewood Cliffs, NJ, 1976.

[42] J. C. Slattery, L. Sagis, E. Oh, Interfacial transport phenomena, Springer, 2006.

[43] E. Kreyszig, Differential geometry, 1991.

[44] J. J. Yoh, X. Zhong, New hybrid Runge-Kutta methods for unsteady reactive flow simulation, AIAA journal 42 (2004) 1593-1600.

[45] Y. Chen, C. Macdonald, The closest point method and multigrid solvers for elliptic equations on surfaces, arXiv preprint arXiv:1307.4354 (2013).

[46] C. H. Gibson, Fine structure of scalar fields mixed by turbulence. i. zero-gradient points and minimal gradient surfaces, Physics of Fluids 11 (1968) 2305.

[47] L. Scriven, Dynamics of a fluid interface equation of motion for Newtonian surface fluids, Chemical Engineering Science 12 (1960) 98-108.

[48] E. Olsson, G. Kreiss, A conservative level set method for two phase flow, Journal of computational physics 210 (2005) 225-246.

[49] R. K. Shukla, C. Pantano, J. B. Freund, An interface capturing method for the simulation of multi-phase compressible flows, Journal of Computational Physics 229 (2010) $7411-7439$.

[50] E. Johnsen, T. Colonius, Implementation of WENO schemes in compressible multicomponent flow problems, Journal of Computational Physics 219 (2006) 715-732.

[51] P. LeFloch, Shock waves for nonlinear hyperbolic systems in nonconservative form, Institute for Math. and its Appl., Minneapolis 593 (1989) 1989.

[52] V. A. Titarev, E. F. Toro, Finite-volume WENO schemes for three-dimensional conservation laws, J. Comput. Phys. 201 (2004) 238-260.

[53] S. Gottlieb, C. Shu, Total variation diminishing Runge-Kutta schemes, Math. Comp 67 (1998) $73-85$. 
[54] V. Titarev, E. Toro, MUSTA schemes for multi-dimensional hyperbolic systems: analysis and improvements, International journal for numerical methods in fluids 49 (2005) 117-147.

[55] E. Toro, V. Titarev, MUSTA fluxes for systems of conservation laws, Journal of Computational Physics 216 (2006) 403-429.

[56] E. F. Toro, Musta: A multi-stage numerical flux, Applied Numerical Mathematics 56 (2006) 1464-1479.

[57] R. P. Fedkiw, T. Aslam, B. Merriman, S. Osher, A non-oscillatory Eulerian approach to interfaces in multimaterial flows (the ghost fluid method), Journal of Computational Physics 152 (1999) 457-492.

[58] Y. Xing, C.-W. Shu, High-order finite volume WENO schemes for the shallow water equations with dry states, Advances in Water Resources 34 (2011) 1026-1038.

[59] M. Dumbser, M. Castro, C. Parés, E. F. Toro, ADER schemes on unstructured meshes for nonconservative hyperbolic systems: Applications to geophysical flows, Computers \& Fluids 38 (2009) 1731-1748.

[60] M. Dumbser, A. Hidalgo, M. Castro, C. Parés, E. F. Toro, FORCE schemes on unstructured meshes II: Non-conservative hyperbolic systems, Computer Methods in Applied Mechanics and Engineering 199 (2010) 625-647.

[61] M. Dumbser, D. S. Balsara, E. F. Toro, C.-D. Munz, A unified framework for the construction of one-step finite volume and discontinuous Galerkin schemes on unstructured meshes, Journal of Computational Physics 227 (2008) 8209-8253.

[62] F. B. Hildebrand, Introduction to numerical analysis, Courier Dover Publications, 1956.

[63] D. Peng, B. Merriman, S. Osher, H. Zhao, M. Kang, A PDE-based fast local level set method, Journal of Computational Physics 155 (1999) 410-438.

[64] F. Memoli, G. Sapiro, S. Osher, Solving variational problems and partial differential equations mapping into general target manifolds, J. Comput. Phys 195 (2002) 263-292.

[65] H. Uddin, R. Kramer, C. Pantano, A Cartesian-based embedded geometry technique with adaptive high-order finite differences for compressible flow around complex geometries, Journal of Computational Physics (2014).

[66] Lawrence Livermore National Laboratory, hypre, high performance preconditioners, http://acts.nersc.gov/hypre/, 2012.

[67] C. Pantano, S. Sarkar, F. A. Williams, Mixing of a conserved scalar in a turbulent reacting shear layer, Journal of Fluid Mechanics 481 (2003) 291-328.

[68] R. Knaus, C. Pantano, On the effect of heat release in turbulence spectra of non-premixed reacting shear layers, Journal of Fluid Mechanics 626 (2009) 67.

[69] P. Lin, M. Bettencourt, S. Domino, T. Fisher, M. Hoemmen, J. Hu, E. Phipps, A. Prokopenko, S. Rajamanickam, C. Siefert, E. Cyr, S. Kennon, A numerical study of reignition induced by a diffusion flame, Submitted to Parallel Processing Letters (2014). 
[70] A. Majda, J. Sethian, The derivation and numerical solution of the equations for zero mach number combustion, Combustion science and technology 42 (1985) 185-205.

[71] M. Heroux, R. Bartlett, R. Howle, V. Hoekstra, J. Hu, T. Kolda, R. Lehoucq, K. Long, R. Pawlowski, E. Phipps, A. Salinger, H. Thornquist, R. Tuminaro, J. Willenbring, A. Williams, An Overview of Trilinos, Technical Report SAND2003-2927, Sandia National Laboratories, 2003.

[72] R. Barlow, J. Frank, 2007. Available at http://www . sandia.gov/TNF/DataArch/FlameD.html.

[73] R. Knaus, A computational approach to flame hole dynamics, Ph.D. thesis, University of Illinois at Urbana-Champaign, 2014.

[74] C. Schneider, A. Dreizler, J. Janicka, E. Hassel, Flow field measurements of stable and locally extinguishing hydrocarbon-fueled jet flames, Combustion and Flame 135 (2003) 185-190.

[75] H. Phillips, Flame in a buoyant methane layer, Symposium (International) on Combustion 10 (1965) 1277 - 1283. Tenth Symposium (International) on Combustion.

[76] R. Daou, J. Daou, J. Dold, Effect of heat loss on flame edges in a non-premixed counterflow within a thermo-diffusive model, Combustion Theory and Modelling 8 (2004) 683 - 699.

[77] C. Shu, S. Osher, Efficient implementation of essentially non-oscillatory shock-capturing schemes, II, Journal of Computational Physics 83 (1989) $32-78$.

[78] J. Shi, C. Hu, C. Shu, A technique of treating negative weights in WENO schemes, Journal of Computational Physics 175 (2002) 108 - 127. 